Pacific

Journal of

Mathematics

THE MASS OF ASYMPTOTICALLY HYPERBOLIC RIEMANNIAN MANIFOLDS

Piotr T. Chruściel and Marc Herzlich

Volume 212 No. 2

December 2003 


\title{
THE MASS OF ASYMPTOTICALLY HYPERBOLIC RIEMANNIAN MANIFOLDS
}

\author{
Piotr T. Chruściel and Marc Herzlich
}

\begin{abstract}
We present a set of global invariants, called "mass integrals", which can be defined for a large class of asymptotically hyperbolic Riemannian manifolds. When the "boundary at infinity" has spherical topology one single invariant is obtained, called the mass; we show positivity thereof. We apply the definition to conformally compactifiable manifolds, and show that the mass is completion-independent. We also prove the result, closely related to the problem at hand, that conformal completions of conformally compactifiable manifolds are unique.
\end{abstract}

\section{Introduction.}

Let $(M, b)$ be a smooth $n$-dimensional Riemannian manifold, $n \geq 2$ and let $\mathcal{N}_{b}$ denote the set of functions $V$ on $M$ such that

$$
\begin{gathered}
\Delta_{b} V+\lambda V=0, \\
\stackrel{\circ}{D}_{i} \stackrel{\circ}{j}_{j} V=V\left(\operatorname{Ric}(b)_{i j}-\lambda b_{i j}\right),
\end{gathered}
$$

for some constant $\lambda<0$. Here $\operatorname{Ric}(b)_{i j}$ denotes the Ricci tensor of the metric $b, \stackrel{\circ}{D}$ the Levi-Civita connection of $b$, and $\Delta_{b}:=b^{k \ell} \stackrel{\circ}{D}_{k} \stackrel{\circ}{D}_{\ell}$ is the Laplacian of $b$. Rescaling $b$ if necessary, we can without loss of generality assume that

$$
\lambda=-n \quad \text { so that } \quad R_{b}=b^{i j} \operatorname{Ric}(b)_{i j}=-n(n-1) .
$$

$(M, b)$ will be called static if

$$
\mathcal{N}_{b} \neq\{0\} .
$$

This terminology is motivated by the fact that for every $V \in \mathcal{N}_{b}$ the Lorentzian metrics defined on $\mathbb{R} \times(M \backslash\{V=0\})$ by the formula

$$
\gamma=-V^{2} d t^{2}+b
$$

are static solution of the Einstein equations, $\operatorname{Ric}(\gamma)=\lambda \gamma$ (and the Riemannian metrics $V^{2} d t^{2}+b$ are actually Einstein as well).

The object of this work is to present a set of global invariants, constructed using $\mathcal{N}_{b}$, for metrics which are asymptotic to a class of static metrics. The model case of interest is the hyperbolic metric, which is static in our sense: The set $\mathcal{N}_{b}$ is then linearly isomorphic to $\mathbb{R}^{n+1}$; however, other classes of 
metrics will also be allowed in our framework. The invariants introduced here stem from a Hamiltonian analysis of general relativity, and part of the work here is a transcription to a Riemannian setting of the Lorentzian analysis in [16]. Related definitions have been given recently by Wang [39] (with a spherical conformal infinity) and Zhang [41] (in dimension 3, again with spherical asymptotic geometry), under considerably more restrictive asymptotic and global conditions. Wang's definition of mass for asymptotically hyperbolic manifolds [39] coincides with ours, when his much stronger asymptotic decay conditions are satisfied (note, however, that his proof of the geometric character of the mass is incomplete, as he ignores the possibility of existence of inequivalent conformal completions). Moreover, the hypothesis of [39] and [41] that $(M, g)$ has compact interior is replaced by that of completeness; this strengthening of the positivity theorem is essential when the associated Lorentzian space-time contains "degenerate" event horizons.

This work is organised as follows: In Section 1 we review a few static metrics, and discuss their properties relevant to the work here. In Section 2 we define the "mass integrals", we make precise the classes of metrics considered, and we show how to obtain global invariants out of the mass integrals. We show that our boundary conditions are sharp, in the sense that their weakening leads to mass integrals which do not provide geometric invariants. It is conceivable that the strengthening of some of our conditions could allow the weakening of some other ones, leading to geometric invariants for other classes of manifolds; we expect that such a mechanism occurs for the Trautman-Bondi mass of asymptotically hyperboloidal manifolds. In several cases of interest one obtains a single invariant, which we call the mass of $(M, g)$, but more invariants are possible depending upon the topology of the "boundary at infinity" $\partial_{\infty} M$ of $M$ - this is determined by the number of invariants of the action of the group of isometries of $b$ on $\mathcal{N}_{b}$, see Section 3 for details. Regardless of this issue, we emphasise that we only consider the "global charges" of [16] related to Killing vectors which are normal to the level sets of $t$ in the space-time metric (0.3): The remaining space-time invariants of [16], associated, e.g., to "rotations" of $M$, involve the extrinsic curvature of the initial data hypersurface and are of no concern in the purely Riemannian setting here. In Section 4 we prove positivity of the mass so obtained for metrics asymptotic to the hyperbolic one. As a corollary of the positivity results we obtain a new uniqueness result for anti-de Sitter space-time, Theorem 4.3. We also consider there the case of manifolds with a compact inner boundary. In Section 5 we show how to define mass for a class of conformally compactifiable manifolds. The question of geometric invariance of the mass is then closely related to the question of uniqueness of conformal completions; in Section 6 we prove that such completions are indeed unique. 


\section{The reference metrics.}

Throughout this paper we will assume that the manifold contains a region $M_{\text {ext }} \subset M$ together with a diffeomorphism

$$
\Phi^{-1}: M_{\text {ext }} \rightarrow[R, \infty) \times N,
$$

where $N$ is a compact boundaryless manifold, such that the reference metric $b$ on $M_{\text {ext }}$ takes the form

$$
\Phi^{*} b=\frac{d r^{2}}{r^{2}+k}+r^{2} \breve{h}=: b_{0},
$$

with $\breve{h}$ - a Riemannian metric on $N$ with scalar curvature $R_{\breve{h}}$ and the constant $k$ equal to

$$
R_{\breve{h}}= \begin{cases}(n-1)(n-2) k, \quad k \in\{0, \pm 1\}, & \text { if } n>2, \\ 0, \quad k=1, & \text { if } n=2,\end{cases}
$$

(recall that the dimension of $N$ is $(n-1)$ ); here $r$ is a coordinate running along the $[R, \infty)$ factor of $[R, \infty) \times N$. There is some freedom in the choice of $k$ in (1.2) when $n=2$, associated with the range of the angular variable $\varphi$ on $N=S^{1}$ (see the discussion in Remark 3.1 below) and we make the choice $k=1$, as it corresponds to the usual form of the two-dimensional hyperbolic space.

When $(N, \breve{h})$ is the unit round $(n-1)$-dimensional sphere $\left(\mathbb{S}^{n-1}, g_{\mathbb{S}^{n-1}}\right)$, then $b$ is the hyperbolic metric. Equations (1.2) and (1.3) imply that the scalar curvature $R_{b}$ of the metric $b$ is constant:

$$
R_{b}=-n(n-1) \text {. }
$$

Moreover the metric $b$ will be Einstein if and only if $\breve{h}$ is. We emphasise that for all our purposes we only need $b$ on $M_{\text {ext }}$, and we continue $b$ in an arbitrary way to $M \backslash M_{\text {ext }}$ whenever required.

The cases of main interest seem to be those where $\breve{h}$ is a space form - it then follows from the results in [16, Appendix B] that we have

$$
\begin{gathered}
k=0,-1 \quad \Longrightarrow \quad \mathcal{N}_{b_{0}}=\operatorname{Vect}\left\{\sqrt{r^{2}+k}\right\} \\
(N, \breve{h})=\left(\mathbb{S}^{n-1}, g_{\mathbb{S}^{n-1}}\right) \quad \Longrightarrow \quad \mathcal{N}_{b_{0}}=\operatorname{Vect}\left\{V_{(\mu)}\right\}_{\mu=0, \ldots, n} \\
V_{(0)}=\sqrt{r^{2}+k}, \quad V_{(i)}=x^{i}
\end{gathered}
$$

with the usual identification of $[R, \infty) \times \mathbb{S}^{n-1}$ with a subset of $\mathbb{R}^{n}$ in (1.6). However, we shall not assume that $\breve{h}$ is a space-form, or that Equations (1.4)(1.6) hold unless explicitly stated.

For the purposes of Section 5 we note the following conformal representation of the metrics (1.2): One replaces the coordinate $r$ by a coordinate $x$ 
defined as

$$
x=\frac{2}{r+\sqrt{r^{2}+k}} \Longleftrightarrow r=\frac{1-k x^{2} / 4}{x},
$$

which brings $b$ into the form

$$
\begin{aligned}
b & =x^{-2}\left(d x^{2}+\left(1-k x^{2} / 4\right)^{2} \breve{h}\right) \\
& =: x^{-2} \widetilde{b},
\end{aligned}
$$

with $\widetilde{b}$ - a metric smooth up to boundary on $\left\{x \in\left[0, x_{R}\right] \times N\right\}$, for a suitable $x_{R}$.

\section{The mass integrals.}

Let $g$ and $b$ be two Riemannian metrics on a manifold $M$, and let $V$ be any function there. We set

$$
e_{i j}:=g_{i j}-b_{i j}
$$

(the reader is warned that the tensor field $e$ here is not a direct Riemannian counterpart of the one in [16]; the latter makes appeal to the contravariant and not the covariant representation of the metric tensor). As before we denote by $\stackrel{\circ}{D}$ the Levi-Civita connection of $b$, and we use the symbol $R_{f}$ to denote the scalar curvature of any metric $f$. The basic identity from which our mass integrals arise is the following:

$$
\sqrt{\operatorname{det} g} V\left(R_{g}-R_{b}\right)=\partial_{i}\left(\mathbb{U}^{i}(V)\right)+\sqrt{\operatorname{det} g}(\rho+Q),
$$

where

$$
\begin{gathered}
\mathbb{U}^{i}(V):=2 \sqrt{\operatorname{det} g}\left(V g^{i[k} g^{j] l} \stackrel{\circ}{D}_{j} g_{k l}+D^{[i} V g^{j] k} e_{j k}\right), \\
\rho:=\left(-V \operatorname{Ric}(b)_{i j}+\stackrel{\circ}{D}_{i} \stackrel{\circ}{D}_{j} V-\Delta_{b} V b_{i j}\right) g^{i k} g^{j \ell} e_{k \ell}, \\
Q:=V\left(g^{i j}-b^{i j}+g^{i k} g^{j \ell} e_{k \ell}\right) \operatorname{Ric}(b)_{i j}+Q^{\prime} .
\end{gathered}
$$

Brackets over a symbol denote anti-symmetrisation, with an appropriate numerical factor $\left(1 / 2\right.$ in the case of two indices). Here $Q^{\prime}$ denotes an expression which is bilinear in $e_{i j}$ and $\stackrel{\circ}{D}_{k} e_{i j}$, linear in $V, d V$ and Hess $V$, with coefficients which are constants in an ON frame for $b$. The idea behind this calculation is to collect all terms in $R_{g}$ that contain second derivatives of the metric in $\partial_{i} \mathbb{U}^{i}$; in what remains one collects in $\rho$ the terms which are linear in $e_{i j}$, while the remaining terms are collected in $Q$; one should note that the first term at the right-hand-side of (2.5) does indeed not contain any terms linear in $e_{i j}$ when Taylor expanded at $g_{i j}=b_{i j}$. The mass integrals will be flux integrals - understood as a limiting process - over the "boundary at infinity of $M$ " of the vector density $\mathbb{U}^{i}$. 
In general relativity a normalising factor $1 / 16 \pi$, arising from physical considerations, is usually thrown in into the definition of $\mathbb{U}^{i}$. From a geometric point of view this seems purposeful when the boundary at infinity is a round two dimensional sphere; however, for other topologies and dimensions, this choice of factor does not seem very useful, and for this reason we do not include it in the definition.

We note that the linearisation of the mass integrands $\mathbb{U}^{i}$ coincides with the linearisation of the charge integrands of $[\mathbf{1 6}]$ evaluated for the Lorentzian metrics ${ }^{4} b=-V^{2} d t^{2}+b,{ }^{4} g=-V^{2} d t^{2}+g$, with $X=\partial_{t}$, on the hypersurface $t=0$; however the integrands do not seem to be identical. Nevertheless, under the conditions of Theorem 2.3 the resulting numbers coincide, because under the asymptotic conditions of Theorem 2.3 only the linearised terms matter.

The convergence of the mass integrals requires appropriate boundary conditions, which are defined using the following orthonormal frame $\left\{f_{i}\right\}_{i=1, n}$ on $M_{\text {ext }}$ :

$$
\Phi_{*}^{-1} f_{i}=r^{-1} \epsilon_{i}, \quad i=1, \ldots, n-1, \quad \Phi_{*}^{-1} f_{n}=\sqrt{r^{2}+k} \partial_{r},
$$

where the $\epsilon_{i}$ 's form an orthonormal frame for the metric $\breve{h}$. We moreover set

$$
g_{i j}:=g\left(f_{i}, f_{j}\right) .
$$

Asymptotic decay conditions 2.1. We shall require:

$$
\begin{aligned}
\int_{M_{\mathrm{ext}}}\left(\sum_{i, j}\left|g_{i j}-\delta_{i j}\right|^{2}+\sum_{i, j, k}\left|f_{k}\left(g_{i j}\right)\right|^{2}\right) r \circ \Phi d \mu_{g}<\infty, \\
\int_{M_{\mathrm{ext}}}\left|R_{g}-R_{b}\right| r \circ \Phi d \mu_{g}<\infty, \\
\exists C>0 \text { such that } C^{-1} b(X, X) \leq g(X, X) \leq C b(X, X) .
\end{aligned}
$$

For the $V$ 's of Equations (1.4) or (1.6) we have

$$
V=O(r), \quad \sqrt{b^{\#}(d V, d V)}=O(r),
$$

where $b^{\#}$ is the metric on $T^{*} M$ associated to $b$, and this behavior will be assumed in what follows:

Proposition 2.2. Let the reference metric $b$ on $M_{\mathrm{ext}}$ be of the form (1.2), suppose that $V$ satisfies (2.10), and assume that $\Phi$ is such that Equations (2.8)-(2.9) hold. Then for all $V \in \mathcal{N}_{b_{0}}$ the limits

$$
H_{\Phi}(V):=\lim _{R \rightarrow \infty} \int_{r=R} \mathbb{U}^{i}\left(V \circ \Phi^{-1}\right) d S_{i}
$$

exist, and are finite. 
The integrals (2.11) will be referred to as the mass integrals.

Proof. For any $R_{1}, R_{2}$ we have

$$
\int_{r=R_{1}} \mathbb{U}^{i} d S_{i}=\int_{r=R_{2}} \mathbb{U}^{i} d S_{i}+\int_{\left[R_{1}, R_{2}\right] \times N} \partial_{i} \mathbb{U}^{i} d^{n} x,
$$

and the result follows from (2.2)-(2.5).

Under the conditions of Proposition 2.2, the integrals (2.11) define a linear map from $\mathcal{N}_{b_{0}}$ to $\mathbb{R}$. Now, each map $\Phi$ used in (1.2) defines in general a different background metric $b$ on $M_{\text {ext }}$, so that the maps $H_{\Phi}$ are potentially dependent upon $\Phi$. (It should be clear that, given a fixed $\breve{h},(2.11)$ does not depend upon the choice of the frame $\epsilon_{i}$ in (2.6).) It turns out that this dependence can be controlled, as follows:

Theorem 2.3. Consider two maps $\Phi_{a}, a=1,2$, satisfying (2.8) together with

$$
\sum_{i, j}\left|g_{i j}-\delta_{i j}\right|+\sum_{i, j, k}\left|f_{k}\left(g_{i j}\right)\right|= \begin{cases}o\left(r^{-n / 2}\right), & \text { if } n>2, \\ O\left(r^{-1-\epsilon}\right), & \text { if } n=2, \text { for some } \epsilon>0 .\end{cases}
$$

Then there exists an isometry $A$ of $b_{0}$, defined perhaps only for $r$ large enough, such that

$$
H_{\Phi_{2}}(V)=H_{\Phi_{1}}\left(V \circ A^{-1}\right) .
$$

Proof. The arguments of the proof of Theorem 2.3 follow closely those given at the beginning of Section 4 and in Section 2 of [16], we need, however, to adapt some of the necessary ingredients to our different setup here. The conclusion of Proposition 5.2 below, which holds for all manifolds $(N, \breve{h})$ considered here, enables us to use Theorem $3.3(2)$ of [16]: If $\Phi_{1}$ and $\Phi_{2}$ are two maps as above satisfying the decay assumptions (2.8)-(2.9) and (2.13) with respect to (isometric) reference metrics $b_{1}:=\left(\Phi_{1}^{-1}\right)^{*} b_{0}$ and $b_{2}:=$ $\left(\Phi_{2}^{-1}\right)^{*} b_{0}$, then there exists an isometry $A$ of the background metric $b_{0}$, defined perhaps only for $r$ large enough, as made clear in Proposition 5.2, such that

$$
\Phi_{2}-\Phi_{1} \circ A=o\left(r^{-n / 2}\right) .
$$

One also has a similar - when appropriately formulated in terms of $b$-orthonormal frames, as in [16] - decay of first two derivatives.

It follows directly from the definition of $H_{\Phi}$ that

$$
H_{\Phi_{1} \circ A}(V)=H_{\Phi_{1}}\left(V \circ A^{-1}\right) \text {. }
$$

In order to establish (2.14) it remains to show that

$$
H_{\Phi_{1} \circ A}(V)=H_{\Phi_{2}}(V) .
$$


Now, Corollary 3.5 of [16] shows that $\Phi_{1} \circ A$ has the same decay properties as $\Phi_{1}$, so that - replacing $\Phi_{1}$ by $\Phi_{1} \circ A$ - to prove (2.15) it remains to consider two maps $\Phi_{1}^{-1}=\left(r_{1}, v_{1}^{A}\right)$ and $\Phi_{2}^{-1}=\left(r_{2}, v_{2}^{A}\right)$ (where $v^{A}$ denote abstract local coordinates on $N$ ) satisfying

$$
\begin{aligned}
& r_{2}=r_{1}+o\left(r_{1}^{1-\frac{n}{2}}\right), \\
& v_{2}^{A}=v_{1}^{A}+o\left(r_{1}^{-\left(1+\frac{n}{2}\right)}\right),
\end{aligned}
$$

together with elements $V_{1}:=V \circ \Phi_{1}^{-1}$ of $\mathcal{N}_{b_{1}}$ and $V_{2}:=V \circ \Phi_{2}^{-1}$ of $\mathcal{N}_{b_{2}}$ having the same expression in the first or the second system of coordinates. Local coordinates $v^{A}$ might not be defined on the whole of $M$; we shall remove this problem by embedding the manifold $N$ in $\mathbb{R}^{2(n-1)}$, so that local coordinates are turned into global coordinates. This has no effect in the sequel of the proof but enables us to consider a well-defined vector field

$$
\zeta=\left(r_{2}-r_{1}\right) \frac{\partial}{\partial r_{1}}+\sum_{A}\left(v_{2}^{A}-v_{1}^{A}\right) \frac{\partial}{\partial v_{1}^{A}},
$$

defined only along $M$, and tangent to $M$. The decay estimates above imply that $\zeta=o\left(r^{-n / 2}\right)$ in the reference metric $b_{1}$; by Theorem $3.3(2)$ of [16] the same holds for its first two $\stackrel{\circ}{D}$-derivatives. Elementary calculations show then that

$$
b_{2}=b_{1}+\mathcal{L}_{\zeta} b_{1}+o\left(r^{-n}\right), \quad V_{2}=V_{1}+\stackrel{\circ}{D}_{i} V \zeta^{i}+o\left(r^{1-n}\right),
$$

together with their first derivatives. Hence, to leading order in powers of $r \approx r_{1}$, everything behaves as if we were considering a first order variation of metrics through the action of the flow of the vector field $\zeta$.

We shall now show that $H_{\Phi_{1}}(V)=H_{\Phi_{2}}(V)$. For the purpose of the calculations that follow it will be easier to replace the local integrand $\mathbb{U}$ by the following one:

$$
\mathbb{U}^{i}=\sqrt{\operatorname{det} b}\left(-V \stackrel{\circ}{D}_{j} g^{i j}+V b^{i j} b_{k l} \stackrel{\circ}{D}_{j} g^{k l}+2 \stackrel{\circ}{D}^{[i} V b^{j] k} e_{j k}\right),
$$

which yields the same limit at infinity when integrated on an element $V$ of $\mathcal{N}_{b}$ on larger and larger spheres (strictly speaking, we should not denote them by the same letter $\mathbb{U}$, since they are different vector densities which give identical results only after an integration process; we shall however do so since expression (2.18) will only be used in the course of the current proof; we emphasise that the definition (2.3) is used in all other places in the paper).

We now compute the variation of $\mathbb{U}$ when passing from the asymptotic map $\Phi_{1}$ (with reference metric $b_{1}$ and function $V_{1}$ ) to the second map $\Phi_{2}$ (with reference metric $b_{2}$ and function $V_{2}$ ). From Equation (2.17), we deduce

$$
\mathbb{U}_{2}^{i}-\mathbb{U}_{1}^{i}=\delta \mathbb{U}^{i}+o\left(r^{1-n}\right)
$$


where $\delta \mathbb{U}^{i}$ is obtained by linearisation in $\zeta$ at $g=b$ and will be computed below, while the remainder terms decay sufficiently fast so that they do not contribute when integrated at infinity against either $b_{1}$ or $b_{2}$. It remains to show that $\delta \mathbb{U}^{i}$ does not contribute either when integrated at infinity. In Equation (2.18), the only terms that contribute a priori to the variation of $\mathbb{U}$ are the following: $b^{i j}, b_{k l}, \sqrt{\operatorname{det} b}, \stackrel{D}{D}$ and $V$, but the decay estimates (2.17) show at first glance that only the variation of $\stackrel{\circ}{D}$ will contribute to the first-order term $\delta \mathbb{U}$. We now compute it using Formulae 1.174 of [8]. In all what follows, we denote $b=b_{1}$ and $V=V_{1}$. Then,

$$
\begin{aligned}
\delta \mathbb{U}^{i}= & \sqrt{\operatorname{det} b}\left(-V \stackrel{\circ}{D}_{k} \stackrel{\circ}{ }^{k} \zeta^{i}+V \stackrel{\circ}{D}_{k} \stackrel{\circ}{D}^{i} \zeta^{k}-2 V \operatorname{Ric}(b)_{k}^{i} \zeta^{k}\right) \\
& +\sqrt{\operatorname{det} b}\left(-2\left(\stackrel{\circ}{D}^{i} V\right) \stackrel{\circ}{D}_{k} \zeta^{k}+\left(\stackrel{\circ}{D}_{k} V\right) \stackrel{\circ}{D}^{i} \zeta^{k}+\left(\stackrel{\circ}{D}^{k} V\right) \stackrel{\circ}{D}_{k} \zeta^{i}\right) .
\end{aligned}
$$

Fortunately, this will appear to be the sum of a divergence term plus lower order terms. The first step is to use the following elementary facts:

$$
\begin{aligned}
-V \stackrel{\circ}{D}_{k} \stackrel{\circ}{D}^{k} \zeta^{i} & =-\stackrel{\circ}{D}_{k}\left(V \stackrel{\circ}{D}^{k} \zeta^{i}\right)+\left(\stackrel{\circ}{D}_{k} V\right) \stackrel{\circ}{D}^{k} \zeta^{i}, \\
V \stackrel{\circ}{D}_{k} \stackrel{\circ}{D}^{i} \zeta^{k} & =\stackrel{\circ}{D}_{k}\left(V \stackrel{\circ}{D}^{i} \zeta^{k}\right)-\left(\stackrel{\circ}{D}_{k} V\right) \stackrel{\circ}{D}^{i} \zeta^{k},
\end{aligned}
$$

which yield

$$
\begin{aligned}
\delta \mathbb{U}^{i}= & 2 \sqrt{\operatorname{det} b}\left(\left(\stackrel{\circ}{D}_{k} V\right) \stackrel{\circ}{D}^{k} \zeta^{i}-\left(\stackrel{\circ}{D}^{i} V\right) \stackrel{\circ}{D}_{k} \zeta^{k}-V \operatorname{Ric}(b)_{k}^{i} \zeta^{k}\right) \\
& + \text { divergence term. }
\end{aligned}
$$

Each of the first two terms in the right-hand side may be transformed with

$$
\begin{gathered}
\left(\stackrel{\circ}{D}_{k} V\right) \stackrel{\circ}{D}^{k} \zeta^{i}=\stackrel{\circ}{D}^{k}\left(\zeta^{i} \stackrel{\circ}{ }^{k} V\right)-\left(\stackrel{\circ}{D}^{k} \stackrel{\circ}{D}_{k} V\right) \zeta^{i}, \\
-\left(\stackrel{\circ}{D}^{i} V\right) \stackrel{\circ}{D}_{k} \zeta^{k}=-\stackrel{\circ}{D}_{k}\left(\zeta^{k} \stackrel{\circ}{D}^{i} V\right)+\left(\stackrel{\circ}{D}_{k} \stackrel{\circ}{D}^{i} V\right) \zeta^{k},
\end{gathered}
$$

and one may also use that $V$ is an element of $\mathcal{N}_{b}$ to conclude that

$$
\mathbb{U}_{2}^{i}-\mathbb{U}_{1}^{i}=\text { divergence term }+o\left(r^{1-n}\right) .
$$

This establishes the covariance of the mass functional.

Remark 2.4. For the purpose of explicit calculations we note that under $(2.13)$ the mass integral $H_{\Phi}\left(V_{(0)}\right)=H_{\Phi}\left(\sqrt{r^{2}+k}\right)$ can be written as:

$$
\begin{aligned}
H_{\Phi}\left(V_{(0)}\right)= & \lim _{R \rightarrow \infty}\left(R^{2}+k\right) \times \\
& \int_{\{r=R\}}\left(-\sum_{i=1}^{n-1}\left\{\frac{\partial e_{i i}}{\partial r}+\frac{k e_{i i}}{r\left(r^{2}+k\right)}\right\}+\frac{(n-1) e_{n n}}{r}\right) d^{n-1} \mu_{h},
\end{aligned}
$$

assuming that the right-hand-side of $(2.25)$ converges. Here $d^{n-1} \mu_{h}$ is the Riemannian measure associated with the metric $h$ induced on the level sets of the function $r$. 
Remark 2.5. Conditions (2.13) are sharp, in the following sense: Let $g$ be the standard hyperbolic metric, thus in a coordinate system $\left(\bar{r}, \bar{v}^{A}\right)$, where the $\bar{v}^{A}$ 's are local coordinates on $\mathbb{S}^{n-1}$, we have

$$
g=\frac{d \bar{r}^{2}}{\bar{r}^{2}+k}+\bar{r}^{2} \breve{h}
$$

Let, for sufficiently large $r, \Phi_{\gamma}^{-1}\left(r, v^{A}\right)=\left(\bar{r}\left(r, v^{A}\right), \bar{v}^{B}\left(r, v^{A}\right)\right)$ be given by the formula

$$
\bar{r}=r+\gamma r^{1-n / 2}, \quad \bar{v}^{A}=v^{A},
$$

where $\gamma$ is a constant. Then $H_{\Phi_{\gamma}}\left(\sqrt{r^{2}+k}\right)$ does depend upon $\gamma$ : In order to see that, consider any background metric of the form

$$
b=a^{2}(r) d r^{2}+r^{2} \breve{h},
$$

and let $g$ satisfy

$$
g=g_{n n}\left(r, v^{A}\right) a^{2}(r) d r^{2}+c\left(r, v^{A}\right) r^{2} \breve{h},
$$

for some differentiable functions $g_{n n}$ and $c$. One finds

$$
\begin{aligned}
\left.\mathbb{U}^{i} d S_{i}\right|_{r=\mathrm{const}}:= & \left.\mathbb{U}^{i} \partial_{i}\right\rfloor\left. d r \wedge d v^{1} \wedge \cdots \wedge d v^{n-1}\right|_{r=\mathrm{const}} \\
= & \frac{(n-1) c^{(n-3) / 2} r^{n-2}}{a(r) \sqrt{g_{n n}}}\left\{V\left(g_{n n}-1-r \frac{\partial c}{\partial r}\right)\right. \\
& \left.\quad+\left(r \frac{\partial V}{\partial r}-V\right)(c-1)\right\} \sqrt{\operatorname{det} \breve{h}_{A B}} d v^{1} \wedge \cdots \wedge d v^{n-1} .
\end{aligned}
$$

Applying this formula to the above $g$ and $b$ one obtains

$$
H_{\Phi_{\gamma}}\left(\sqrt{r^{2}+k}\right)=\frac{1}{4}(n+8) n(n-1) \gamma^{2} \operatorname{Vol}_{g_{\mathbb{S} n-1}}\left(\mathbb{S}^{n-1}\right) .
$$

One can also check that the numerical value of the linearised expression (2.25) reproduces the right-hand-side of (2.30) for the metrics at hand, thus is again not invariant under (2.27).

\section{The mass.}

In the asymptotically flat case the mass is a single number which one assigns to each end of $M$ ([4] and [15]); it is then natural to enquire whether there are some geometrically defined numbers one can extract out of the family of maps $H_{\Phi}$. This will depend upon the structure of $\mathcal{N}_{b_{0}}$ and we shall give here a few important examples. Throughout this section we assume that $\mathcal{N}_{b_{0}} \neq \emptyset$.

A. The simplest case is that of the manifold $(N, \breve{h})$ of (1.1)-(1.2) having a strictly negative Ricci tensor, with scalar curvature $R_{\breve{h}}=-(n-1)(n-2)$, 
so that $n \geq 3$ and $k=-1$ in (1.2). Similarly to the space forms discussed in Section $1, \mathcal{N}_{b_{0}}$ is then [16, Appendix B] one dimensional:

$$
V \in \mathcal{N}_{b_{0}} \Longleftrightarrow V=\lambda V_{(0)}, \lambda \in \mathbb{R}, \quad V_{(0)}:=\sqrt{r^{2}+k} .
$$

The coordinate system of (1.2) is uniquely defined, so is the function $V_{(0)}$; the number

$$
m:=H_{\Phi}\left(V_{(0)}\right),
$$

calculated using any $\Phi$ satisfying the conditions of Theorem 2.3, provides the desired, $\Phi$-independent definition of mass relative to $b_{0}$, whenever (2.6) and (2.13) hold.

B. Consider, next, the case of a flat $(N, \breve{h})$ with $n \geq 3$, so that $k=0$ in (1.2). Equation (3.1) holds again; however, the coordinate $r$ is not anymore uniquely defined by $b$, since (1.2) is invariant under the rescalings

$$
r \rightarrow a r, \quad \breve{h} \rightarrow a^{-2} \breve{h}, \quad a \in \mathbb{R}^{*} .
$$

This freedom can be gotten rid of by requiring, e.g.,

$$
\operatorname{Vol}_{\breve{h}}(N)=1 ;
$$

the number $m$ obtained then from (3.2), with $V_{(0)}$ as in (3.1), provides the desired invariant.

C. The case $k=+1$ requires more work. Consider first the case where $(N, \breve{h})=\left(\mathbb{S}^{n-1}, g_{\mathbb{S}^{n-1}}\right)$, so that the reference metric is the hyperbolic metric; it is convenient to start with a discussion of $\mathcal{N}_{b}$ in two models of the hyperbolic space: In the ball model, we consider the ball $\mathbb{B}=\left\{x \in \mathbb{R}^{n},|x|<1\right\}$ endowed with the metric $b=\omega^{-2} \delta$, where

$$
\omega=\frac{1}{2}\left(1-|x|^{2}\right)
$$

and $\delta$ is the flat Euclidean metric. From (1.5)-(1.6) one finds that the set $\mathcal{N}_{b}$ defined in (0.1)-(0.2) is the $(n+1)$-dimensional vector space spanned by the following basis of functions:

$$
V_{(0)}=\frac{1+|x|^{2}}{1-|x|^{2}}, \quad V_{(i)}=\frac{2 x^{i}}{1-|x|^{2}},
$$

where $x^{i}$ is any of the Cartesian coordinates on the flat ball. In geodesic coordinates around an arbitrary point in the hyperbolic space, the hyperbolic metric is $b=d r^{2}+\sinh ^{2}(r) g_{\mathbb{S}^{n-1}}$ and the above orthonormal basis of $\mathcal{N}_{b}$ may be rewritten as $V_{(0)}=\cosh (r)$ and $V_{(i)}=\sinh (r) n^{i}$, where $n^{i}$ is the restriction of $x^{i}$ to the unit sphere centred at the pole.

The space $\mathcal{N}_{b}$ is naturally endowed with a Minkowski metric $\eta$, with signature $(+,-, \cdots,-)$, issued from the action of the group of isometries $O^{+}(n, 1)$ of the hyperbolic metric ( cf., e.g., [16, Appendix B]). The basis given above 
is then orthonormal with respect to this metric, with the vector $V_{(0)}$ being timelike, i.e., $\eta\left(V_{(0)}, V_{(0)}\right)>0$. We define the time orientation of $\mathcal{N}_{b}$ using this basis - by definition a timelike vector $X^{(\mu)} V_{(\mu)}$ is future directed if $X^{(0)}>0$, similarly for covectors; this finds its roots in a Hamiltonian analysis in the associated Lorentzian space-time. Assuming that there exists a map $\Phi$ for which the convergence conditions of Proposition 2.2 are satisfied, we set

$$
p_{(\mu)}:=H_{\Phi}\left(V_{(\mu)}\right) .
$$

Under isometries of $b$ the $V_{(\mu)}$ 's are reshuffled amongst each other under the usual covariant version of the defining representation of the Lorentz group $O^{+}(n, 1)$. It follows that the number

$$
m^{2}:=\left|\left(p_{(0)}\right)^{2}-\sum_{i=1}^{n}\left(p_{(i)}\right)^{2}\right|
$$

is a geometric invariant, which provides the desired notion of mass for a spherical asymptotic geometry. The nature of the action of $O^{+}(n, 1)$ on $\mathcal{N}_{b}$ shows that the only invariants which can be extracted out of the $H_{\Phi}$ 's are $m^{2}$ together with the causal character of $p_{(\mu)}$ and its future/past pointing nature if relevant. Under natural geometric conditions $p_{(\mu)}$ is timelike future pointing or vanishing, see Section 4 below. For timelike $p_{(\mu)}$ 's it appears natural to choose the sign of $m$ to coincide with that of $p_{(0)}$, and this is the choice we shall make.

Suppose, finally, that the manifold $(N, \breve{h})$ is the quotient of the unit round sphere $\left(\mathbb{S}^{n-1}, g_{\mathbb{S}^{n-1}}\right)$ by a subgroup $\Gamma$ of its group of isometries. For generic $\Gamma$ 's one expects the conformal isometry group of $(N, \breve{h})$ to be trivial, in which case all the integrals $p_{(\mu)}$ defined by Equation (3.5) define invariants. In any case, for nontrivial $\Gamma$ 's conformal isometries of $(N, \breve{h})$ are isometries (for compact Einstein manifolds which are not round spheres the group of conformal isometries coincides with the group of isometries; this follows immediately, e.g., from what is said in [29]; P.T.C. is grateful to A. Zeghib and C. Frances for useful comments concerning the structure of the group of conformal isometries of quotients of spheres), and, in addition to $m, p_{(0)}$ becomes then a geometric invariant. Further invariants may occur depending upon the details of the action of the group of isometries of $(N, \breve{h})$ on $\mathcal{N}_{b_{0}}$.

Remark 3.1. Our results also apply in dimension $n=2$. This might seem somewhat surprising at first sight, because there is no direct useful equivalent of asymptotic flatness and of the associated notion of mass given by an ADM-type integral in dimension 2: When the scalar curvature is in $L^{1}$, the appropriate analogue of mass is the deficit angle, as made precise by the Shiohama theorem [37]. For the metrics considered here the Shiohama theorem does not apply; however, the metrics we study can be thought of as 
having a minus infinite deficit angle, consistently with a naively understood version of the Shiohama theorem - the ratio of the length of distance circles to the distance from any compact set tends to infinity as the distance does. Examples of metrics on $M_{\text {ext }}$ which satisfy our asymptotic conditions and have a well-defined nontrivial mass - with respect to a background given by the 2-dimensional hyperbolic metric - are provided by the Riemannian counterpart of the generalised $(2+1)$-dimensional Kottler metrics,

$$
g=\frac{d r^{2}}{r^{2}-\eta}+r^{2} d \varphi^{2}, \quad \varphi \in[0,2 \pi] \bmod 2 \pi,
$$

for some constant $\eta \in \mathbb{R} ; \eta=-1$ corresponds to the standard hyperbolic metric (as pointed out by Bañados, Teitelboim and Zanelli [3], for positive $\eta$ the associated static Lorentzian space-times with $V=\sqrt{r^{2}-\eta}$ can be extended to space-times containing a black-hole region). The metrics (3.7) have constant Gauss curvature equal to minus one for all $\eta \in \mathbb{R}$, so the integral condition on $R_{g}$ in (2.8) holds with

$$
b=\frac{d r^{2}}{r^{2}+1}+r^{2} d \varphi^{2}
$$

the remaining conditions arising from (2.8), as well as (2.9) and (2.13), are easily checked. Applying formula (2.25) one obtains

$$
m=p_{(0)}=2 \pi(1+\eta)
$$

(the remaining $p_{(\mu)}$ 's are zero by symmetry considerations). For strictly negative $\eta$ there is a sense in which $m$ is related to a deficit angle, as follows: A coordinate transformation $r \rightarrow \lambda r, \varphi \rightarrow \varphi / \lambda$, with $\lambda^{2}=-\eta$ brings the metric (3.7) to the standard hyperbolic space form,

$$
g=\frac{d r^{2}}{r^{2}+1}+r^{2} d \varphi^{2}, \quad \varphi \in[0,2 \pi / \lambda] \bmod 2 \pi / \lambda,
$$

except for the changed range of variation of the angular variable $\varphi$; that range will coincide with the standard one if and only if the mass vanishes. For metrics asymptotic to (3.7) the geometric invariance of the mass should follow directly from this deficit angle character. This suggests strongly that some methods specific to dimension 2, perhaps in the spirit of the Shiohama theorem ( $c f$. also [32]), could provide simpler proofs of geometric invariance and positivity when $n=2$; we have not investigated this issue any further.

\section{Mass positivity for metrics asymptotic to the standard hyperbolic metric.}

In this section we consider metrics asymptotic to the standard hyperbolic metric $b$ of constant negative curvature -1 ; by this we mean that the Riemannian manifold $(N, \breve{h})$ is the unit round sphere $\left(\mathbb{S}^{n-1}, g_{\mathbb{S}^{n-1}}\right)$. We wish 
to show that the usual positivity theorem holds under the weak asymptotic hypotheses considered in the previous sections.

Theorem 4.1. Let $(M, g)$ be a complete boundaryless spin manifold with a $C^{2}$ metric, and with scalar curvature satisfying

$$
R_{g} \geqslant-n(n-1) \text {, }
$$

and suppose that the asymptotic conditions (2.8) and (2.13) hold with $(N, \breve{h})$ $=\left(\mathbb{S}^{n-1}, g_{\mathbb{S}^{n-1}}\right)$. Then the covector $p_{(\mu)}$ defined by Equation (3.5) is timelike future directed or zero (in particular $p_{(0)} \geq 0$ ). Moreover, it vanishes if and only if $(M, g)$ is isometrically diffeomorphic to the hyperbolic space.

Remark 4.2. 1 . The $C^{2}$ differentiability of the metric can be replaced by a weighted $W^{2, p}$ Sobolev condition.

2. As already pointed out, we say that a linear functional $p$ on $\mathcal{N}_{b}$ is causal (resp. timelike) and future-directed if it can be written as $\left(p_{(0)}, \ldots, p_{(n)}\right)$ in any orthonormal and future-oriented basis $\left(V_{(0)}, \ldots, V_{(n)}\right)$ with

$$
\left(p_{(0)}\right)^{2}-\sum_{i=1}^{n}\left(p_{(i)}\right)^{2} \geqslant 0 \text { and } p_{(0)} \geqslant 0 \quad(\text { resp. }>0) \text {. }
$$

This is the obvious equivalent of the corresponding definition for vectors; note, however, that with our signature $(-,+, \ldots,+)$ future directed vectors are not mapped to future directed covectors by the isomorphism of $T M$ with $T^{*} M$ associated with the metric.

We emphasise that the Lorentz vector character of $p_{(\mu)}$ is not related to the tangent space of some point of $M$, or of some "abstract asymptotic point" ("the tangent space at $i^{o}$ " — this last interpretation can be given to energy-momentum in the asymptotically Euclidean context), but arises from the fact that the adjoint action of the isometry group of the (standard) hyperbolic space, on the subspace of its Lie algebra singled out by Equations (0.1)-(0.2), is that of the defining representation of the Lorentz group on $\mathbb{R}^{n+1}$.

3. Condition (2.8b) is actually not necessary for positivity, in the following sense: Under the remaining conditions of Theorem 4.1, the argument of the proof of Proposition 2.2 shows that $p_{(0)}=\infty$ whenever $(2.8 \mathrm{~b})$ does not hold.

4. Such a theorem cannot be obtained in a more general setting. For instance, in the asymptotically Euclidean context, it is well-known that positivity statements may fail if the metric is asymptotic to some $\mathbb{Z}_{2}$-quotient of the Euclidean space [30]. In the asymptotically hyperbolic setting, Horowitz and Myers [25] have constructed an infinite family of metrics with ends asymptotic to a cuspidal hyperbolic metric (the topology of the end is $\mathbb{R} \times T^{2}$ ), and with masses as negative as desired. If the topology of the end is the product of a half-line with a negatively curved Riemann surface, the mass 
may also be negative when minimal interior boundaries are allowed, and it is expected that the infimum of the possible masses is achieved only for the Kottler black-hole metrics $[\mathbf{2 5}, \mathbf{2 8}, \mathbf{3 8}, \mathbf{1 2}]$ and $[\mathbf{1 7}]$.

5. The $C^{2}$ differentiability of the metric can be replaced by weighted Sobolev-type conditions; this is, however, of no concern to us here.

As a corollary of Theorem 4.1, together with [17, Theorem I.3] and the remarks at the end of Section V of [17] one has (see [17, Corollary I.4]; compare [9]):

Theorem 4.3. Let $V$ be a strictly positive function on a three dimensional manifold $M$ such that the metric

$$
\gamma:=-V^{2} d t^{2}+g
$$

is a static solution of the vacuum Einstein equations with strictly negative cosmological constant on the space-time $\mathcal{M}:=\mathbb{R} \times M$. If:

(i) $(M, g)$ is $C^{3}$ compactifiable in the sense of Section 5 below, and if

(ii) the conformal boundary at infinity of $M$ is $S^{2}$, with $V^{-2} g$ extending by continuity to the unit round metric on $S^{2}$,

then $(M, g)$ is the hyperbolic space, so that $(\mathcal{M}, \gamma)$ is the anti-de Sitter spacetime.

Preliminaries to the proof. The Proof of Theorem 4.1 will follow the Gibbons-Hawking-Horowitz-Perry variation [21] of the classical Witten argument for the positivity of mass [40] (cf. also [34], [1] and the remarks done in [18]), and relies on the existence on the hyperbolic space of a wealth of distinguished spinor fields, called imaginary Killing spinors. These are solutions $\varphi$ of the differential equation

$$
\widehat{D}_{X}^{b} \varphi=D_{X}^{b} \varphi+\frac{i}{2} c_{b}(X) \varphi=0,
$$

where we denote by $c_{b}(X) \varphi$ the Clifford action of a vector $X$ on a spinor $\varphi$ with respect to the metric $b$. On hyperbolic space there is a set of maximal dimension of imaginary Killing spinors, which trivialise the spinor bundle. They can be described explicitly in the following manner [6]: One may choose the standard basis $\left\{\partial_{i}\right\}$ of the flat space as reference frame, thus inducing an isomorphism between the spinor frame bundle of $(\mathbb{B}, e)$ and $\mathbb{B} \times \operatorname{Spin}(n)$. This can be transferred to the hyperbolic space through the usual conformal covariance (of "weight zero") of spinor bundles [20]. In this trivialisation, the Killing spinors of $b$ are then the spinor fields $\varphi_{u}$ given by

$$
\varphi_{u}(x)=\omega^{-\frac{1}{2}}\left(1-i c_{\delta}(x)\right) u
$$

where $u$ is any nonzero constant spinor on the flat ball $\mathbb{B}$, and $\omega$ is the conformal factor of the hyperbolic metric defined in (3.3). 
Following the terminology due to H. Baum, Th. Friedrich and I. Kath $[\mathbf{1 9}$, 7] and [6], the spinor $\varphi_{u}$ is said to be of Type I (resp. of Type II) if

$$
\|u\|_{\delta}^{4}+\sum_{i=1}^{n}\left\langle c_{\delta}\left(\partial_{i}\right) u, u\right\rangle_{\delta}^{2} \text { is zero (resp. is positive). }
$$

Type I spinors are actually sufficient for our purposes, we shall describe these ones only. For any imaginary Killing spinor, the function

$$
V_{u}=\left\langle\varphi_{u}, \varphi_{u}\right\rangle_{b}
$$

is always an element of $\mathcal{N}_{b}$. If $\varphi_{u}$ is moreover of Type I, then there is a set of $n$ constants $\left(a_{i}\right) \in \mathbb{S}^{n-1} \subset \mathbb{R}^{n}$ and a constant $\lambda>0$, such that $V_{u}=\lambda\left(V_{(0)}-\sum_{i} a_{i} V_{(i)}\right)$ : Indeed, an explicit computation from Equation (4.3) above shows that, in the ball model,

$$
V_{u}(x)=\|u\|_{\delta}^{2} \frac{1+|x|^{2}}{1-|x|^{2}}+i \sum_{j=1}^{n}\left\langle c_{\delta}\left(\partial_{j}\right) u, u\right\rangle_{\delta} \frac{2 x^{j}}{1-|x|^{2}},
$$

which is clearly future directed, and the Type I condition (4.4) yields that $V_{u}$ is isotropic in $\mathcal{N}_{b}$. This shows in particular that Killing spinors of Type I always exist on the hyperbolic space in any dimension. Further, e.g., as a result of covariance under isometries, any future directed isotropic combination $V_{(0)}-\sum_{i} a_{i} V_{(i)},\left(a_{i}\right) \in \mathbb{S}^{n-1}$, can be obtained as a $V_{u}$ for some Type I Killing spinor (i.e., for some constant spinor $u$ on the flat ball) in any dimension. For later use we also note that

$$
d V_{u}(X)=i\left\langle c_{b}(X) \varphi_{u}, \varphi_{u}\right\rangle_{b} .
$$

Proof of Theorem 4.1. Let $A$ be the symmetric endomorphism defined over $M_{\text {ext }}$ by $g(A \cdot, A \cdot)=b(\cdot, \cdot)$, which we will take to be of the form

$$
A=I-\frac{1}{2} e+\{\text { quadratic and higher order in } e\}
$$

if $e$ is small enough; by this we mean that $A^{i}{ }_{j}=\delta_{j}^{i}-\frac{1}{2} b^{i k} e_{k j}+$ a second order Taylor expansion error term. One may use $A$ as an isomorphism between the orthonormal frame bundles of $b$ and $g$ and any lift of it as an isomorphism between their spinor frame bundles. This enables, as in [1] (compare [10]), to transfer the spin connection $D^{b}$ of $\left(M_{\text {ext }}, b\right)$ on the spinor bundle of $\left(M_{\mathrm{ext}}, g\right)$; for notational convenience, the new connection will be denoted by $D^{\hat{b}}$. Note that this has the effect that the Clifford action $c_{b}(X)$ of a vector $X$ is transformed into the Clifford action $c_{g}(A X)$ of $A X$. As a consequence, the transferred spinors, still denoted by $\varphi_{u}$, are now solutions of

$$
\widehat{D}_{X}^{\hat{b}} \varphi_{u}=D_{X}^{\hat{b}} \varphi_{u}+\frac{i}{2} c_{g}(A X) \varphi_{u}=0 .
$$


We now denote by $D$ the spinor connection associated to the Levi-Civita connection of the metric $g$ and define the modified connection on spinors

$$
\widehat{D}_{X}=D_{X}+\frac{i}{2} c_{g}(X) \text {. }
$$

For any $\varphi_{u}$ we set

$$
\Phi_{u}=\chi \varphi_{u}+\psi_{u},
$$

where $\chi$ is a cut-off function that vanishes outside of $M_{\text {ext }}$ and is equal to 1 for $r$ large enough. Suppose, first, that $\psi_{u}$ is compactly supported, hence vanishes for $r \geq R$ for some $R$ on $M_{\text {ext }}$. We apply the standard SchrödingerLichnerowicz ([33] and [36]) formula relating the rough Laplacian of the modified connection $\widehat{D}$ to the Dirac Laplacian $\widehat{\mathfrak{D}} * \widehat{\mathfrak{D}}[\mathbf{1}]$, where

$$
\widehat{\mathfrak{D}} \Phi_{u}=\mathfrak{D} \Phi_{u}-\frac{n i}{2} \Phi_{u}
$$

with $\mathfrak{D}$ being the usual Dirac operator associated with the metric $g$. Letting $S_{R}=\{r=R\} \subset M_{\text {ext }}$ one obtains

$$
\begin{aligned}
& \int_{M \backslash\{r \geq R\}}\left\|\widehat{D} \Phi_{u}\right\|_{g}^{2}+\frac{1}{4}\left(R_{g}+n(n-1)\right)\left\|\Phi_{u}\right\|_{g}^{2}-\left\|\widehat{\mathfrak{D}} \Phi_{u}\right\|_{g}^{2} \\
& =\int_{S_{R}} B_{A \nu}\left(\Phi_{u}\right) \\
& =\int_{S_{R}} B_{A \nu}\left(\varphi_{u}\right),
\end{aligned}
$$

where $\nu$ is the outer $b$-unit normal to $S_{r}$, so that $A \nu$ is its outer $g$-unit normal, and $B_{A \nu}\left(\varphi_{u}\right)$ is the boundary integrand, explicitly defined by

$$
B_{Y}(\rho)=\left\langle\widehat{D}_{Y} \rho+c_{g}(Y) \widehat{\mathfrak{D}} \rho, \rho\right\rangle_{g}
$$

for any spinor $\rho$ and vector $Y$.

Assume that $(M, g)$ is not the hyperbolic space, otherwise there is nothing to prove. Let $H$ be the usual Hilbertian completion of the space of compactly supported smooth spinors $\psi$ on $M$ with respect to the norm defined as

$$
\|\psi\|_{H}^{2}:=\int_{M}\left(\|\widehat{D} \psi\|_{g}^{2}+\frac{1}{4}\left(R_{g}+n(n-1)\right)\|\psi\|_{g}^{2}\right) d \mu_{g} .
$$

We wish to show that for any $\Phi_{u}=\chi \varphi_{u}+\psi_{u}$, with $\psi_{u} \in H$, we will have

$$
\int_{M}\left\|\widehat{D} \Phi_{u}\right\|_{g}^{2}+\frac{1}{4}\left(R_{g}+n(n-1)\right)\left\|\Phi_{u}\right\|_{g}^{2}-\left\|\widehat{\mathfrak{D}} \Phi_{u}\right\|_{g}^{2}=\lim _{R \rightarrow \infty} \int_{S_{R}} B_{A \nu}\left(\varphi_{u}\right) .
$$

We start by showing that $H$ can be identified with a space $\mathcal{H}$ of $H_{\text {loc }}^{1}$ spinor fields on $M$, with the norm $\|\cdot\|_{H}$ still given by (4.10) (after identification) for all $\psi \in H$. First, it is not too difficult to show [5] that in dimension 
larger than or equal to three there exists a strictly positive $L_{\text {loc }}^{\infty}$ function $w$ on $M$ such that for all $H_{\text {loc }}^{1}$ spinor fields $\psi$ with compact support we have

$$
\int_{M}\|\psi\|_{g}^{2} w d \mu_{g} \leq \int_{M}\|\widehat{D} \psi\|_{g}^{2} d \mu_{g}
$$

The function $w$ can be chosen to be constant in the asymptotically hyperbolic end. In dimension two one can also prove (4.12) if one assumes further that there are no imaginary Killing spinors. This is sufficient for our purposes because, if there exists a Killing spinor then, by [6], we are in hyperbolic space, where there is nothing to prove. So one might as well suppose that there are no such spinors.

Let $\mathcal{H}$ be the space of measurable spinor fields on $M$ such that

$$
\|\psi\|_{\mathcal{H}}^{2}:=\int_{M}\|\psi\|_{g}^{2}\left(w+\frac{1}{4}\left(R_{g}+n(n-1)\right)\right) d \mu_{g}+\int_{M}\|\widehat{D} \psi\|_{g}^{2} d \mu_{g}<\infty
$$

where $\widehat{D} \psi$ is understood in the distributional sense. Define $\mathcal{H} \subset \mathcal{H}$ as the completion of $C_{c}^{\infty}$, in $\mathcal{H}$, with respect to the $\|\cdot\|_{\mathcal{H}}$ norm. It is then easy to verify the following:

Proposition 4.4. The inequality (4.12) remains true for all $\psi \in \mathcal{H}$.

Proof. Both sides of (4.12) are continuous on $\left(\mathcal{H},\|\cdot\|_{\mathcal{H}}\right)$.

Proposition 4.5. If $(M, g)$ is complete then $\mathcal{H}=\mathcal{H}$.

Proof. If $\phi \in \mathcal{H}$ then the sequence $\chi_{i} \phi$ converges to $\phi$ in $\left(\mathcal{H},\|\cdot\|_{\mathcal{H}}\right)$, where $\chi_{i}(p)=\chi\left(d_{p_{0}}(p) / i\right)$, where $d_{p_{0}}$ is the distance to some chosen point $p_{0} \in M$, while $\chi: \mathbb{R} \rightarrow[0,1]$ is a smooth function such that $\left.\chi\right|_{[0,1]}=1,\left.\chi\right|_{[2, \infty)}=0$. Smoothing $\chi_{i} \phi$ using the usual convolution operator yields the result.

Proposition 4.6. If $(M, g)$ is complete then there is a natural continuous bijection between $\left(\mathcal{H},\|\cdot\|_{\mathcal{H}}\right)$ and $\left(H,\|\cdot\|_{H}\right)$ which is the identity on $C_{c}^{1}$; in particular, elements of $H$ can be identified with spinor fields on $M$ which are in $\mathcal{H}$.

Proof. By Proposition 4.5 both spaces are Hilbert spaces containing $C_{c}^{1}$ as a dense subspace, with the norms being equivalent when restricted to $C_{c}^{1}$ by Proposition 4.4.

Let $F(\psi)$ denote the left-hand side of Equation (4.11) with $\Phi_{u}=\chi \varphi_{u}+\psi$ there, let $\psi_{i} \in C_{c}^{1}$ converge to $\psi$ in $H$, we have 


$$
\begin{aligned}
F(\psi)-F\left(\psi_{i}\right)= & \|\psi\|_{H}^{2}-\left\|\psi_{i}\right\|_{H}^{2} \\
& +2 \int_{M}\left\langle\widehat{D}\left(\chi \varphi_{u}\right), \widehat{D}\left(\psi-\psi_{i}\right)\right\rangle \\
& -2 \int_{M}\left\langle\widehat{\mathfrak{D}}\left(\chi \varphi_{u}\right), \widehat{\mathfrak{D}}\left(\psi-\psi_{i}\right)\right\rangle \\
& +\frac{1}{2} \int_{M}\left(R_{g}+n(n-1)\right)\left\langle\chi \varphi_{u}, \psi-\psi_{i}\right\rangle .
\end{aligned}
$$

It should be clear from the fact that $\widehat{D}\left(\chi \varphi_{u}\right) \in L^{2}(M)$ that all the terms above converge to zero as $i$ tends to infinity, except perhaps for the last one (recall that we are only assuming that $\left.0 \leq\left(R_{g}+n(n-1)\right)|V| \in L^{1}\left(M_{\text {ext }}\right)\right)$; the convergence of that last term can be justified as follows:

$$
\begin{aligned}
& \left|\int_{M}\left(R_{g}+n(n-1)\right)\left\langle\chi \varphi_{u}, \psi-\psi_{i}\right\rangle\right| \\
& \leq\left(\int_{M}\left(R_{g}+n(n-1)\right)\left\|\chi \varphi_{u}\right\|_{g}^{2}\right)^{1 / 2}\left(\int_{M}\left(R_{g}+n(n-1)\right)\left\|\psi-\psi_{i}\right\|_{g}^{2}\right)^{1 / 2} \\
& \leq\left\|\chi \varphi_{u}\right\|_{H}\left\|\psi-\psi_{i}\right\|_{H} .
\end{aligned}
$$

(Here we have applied the Cauchy-Schwarz inequality associated with the positive quadratic form occurring in the left-hand-side above.) Now, $F\left(\psi_{i}\right)=$ $F(0)$, and we have shown that Equation (4.11) holds for all $\psi_{u} \in H$, as claimed.

To obtain positivity of the left-hand-side of Equation (4.11) we seek a $\Phi_{u}$ such that

$$
\widehat{\mathfrak{D}} \Phi_{u}=0 \Longleftrightarrow \widehat{\mathfrak{D}} \psi_{u}=-\widehat{\mathfrak{D}}\left(\chi \varphi_{u}\right) .
$$

We now use the fact that $\varphi_{u}$ solves (4.6), hence

$$
\widehat{D}_{X} \varphi_{u}=\left(D_{X}-D_{X}^{\hat{b}}\right) \varphi_{u}-\frac{i}{2} c_{g}(A X-X) \varphi_{u} .
$$

Now, in any $g$-orthonormal frame $\left\{f_{\alpha}\right\}_{\alpha=1, \ldots, n}$, if $\omega$ denotes the connection 1-forms of either $D$ or $D^{\hat{b}}$ (with the obvious notations), one has:

$$
D_{X}-D_{X}^{\hat{b}}=\frac{1}{4} \sum_{\alpha, \beta=1}^{n}\left(\omega_{\alpha \beta}(X)-\omega_{\alpha \beta}^{\hat{b}}(X)\right) c_{g}\left(f_{\alpha}\right) c_{g}\left(f_{\beta}\right) \text {. }
$$

The calculations of $[\mathbf{1}$, Section 2.2] lead then to

$$
\left|\widehat{\mathfrak{D}} \varphi_{u}\right| \leq C\left(|\stackrel{\circ}{D} A|_{b}+|A-\mathrm{id}|_{b}\right)\left|\varphi_{u}\right| \quad \Longrightarrow \quad \widehat{\mathfrak{D}}\left(\chi \varphi_{u}\right) \in L^{2}\left(M, d \mu_{g}\right) .
$$

Equation (4.17) and arguments known in principle $[\mathbf{1}, \mathbf{3 5}, \mathbf{1 4}]$ and $[\mathbf{2 3}]$ (cf. also [5]) show that there exists $\psi_{u}$ in $H$ such that Equation (4.14) 
holds. It then remains to show that the integral at the right-hand-side of Equation (4.11) is related to the map $H_{\Phi}$. In order to do this, we complement $\nu$ into a direct $b$-orthonormal basis $\left\{\nu, e_{i}\right\}_{i=1, \ldots, n-1}$. Seen as sitting in $M$, $\left\{A \nu, A e_{i}\right\}_{i=1, \ldots, n-1}$ is a direct $g$-orthonormal basis on $S_{r}$. One easily finds

$$
B_{A \nu}\left(\varphi_{u}\right)=\sum_{i=1}^{n-1}\left\langle c_{g}(A \nu) c_{g}\left(A e_{i}\right) \widehat{D}_{A e_{i}} \varphi_{u}, \varphi_{u}\right\rangle_{g}
$$

From (4.15) and (4.16), we obtain

$$
\begin{aligned}
B_{A \nu}\left(\varphi_{u}\right)= & \frac{1}{4} \sum_{i=1}^{n-1} \sum_{\alpha, \beta=1}^{n}\left(\omega_{\alpha \beta}\left(A e_{i}\right)\right. \\
& \left.-\omega_{\alpha \beta}^{\hat{b}}\left(A e_{i}\right)\right)\left\langle c_{g}(A \nu) c_{g}\left(A e_{i}\right) c_{g}\left(f_{\alpha}\right) c_{g}\left(f_{\beta}\right) \varphi_{u}, \varphi_{u}\right\rangle \\
+ & \frac{i}{2} \sum_{i=1}^{n-1}\left\langle c_{g}(A \nu) c_{g}\left(A e_{i}\right) c_{g}\left(A\left(A e_{i}\right)-A e_{i}\right) \varphi_{u}, \varphi_{u}\right\rangle \\
= & -\frac{1}{2} \sum_{i, j=1}^{n-1}\left(\omega_{j 0}\left(A e_{i}\right)-\omega_{j 0}^{\hat{b}}\left(A e_{i}\right)\right)\left\langle c_{g}\left(A e_{i}\right) c_{g}\left(A e_{j}\right) \varphi_{u}, \varphi_{u}\right\rangle \\
+ & \frac{1}{4} \sum_{i, j, k=1}^{n-1}\left(\omega_{j k}\left(A e_{i}\right)\right. \\
& \left.-\omega_{j k}^{\hat{b}}\left(A e_{i}\right)\right)\left\langle c_{g}(A \nu) c_{g}\left(A e_{i}\right) c_{g}\left(A e_{j}\right) c_{g}\left(A e_{k}\right) \varphi_{u}, \varphi_{u}\right\rangle \\
+ & \frac{i}{2} \sum_{i=1}^{n-1}\left\langle c_{g}(A \nu) c_{g}\left(A e_{i}\right) c_{g}\left(A\left(A e_{i}\right)-A e_{i}\right) \varphi_{u}, \varphi_{u}\right\rangle,
\end{aligned}
$$

where the subscript ${ }_{0} 0$ in the last formula denotes the basis element $A \nu$. These formulae correct Equation (34), page 20, in [1]: In the second line of that equation the multiplicative factor $1 / 4$ should be changed to $1 / 8$; this minor mistake carries over to all the equations that follow.

One may now use $[\mathbf{1}$, Formulae (2-3)] to compute the difference between the connection 1-forms of $D$ and $D^{b}$ (or, equivalently, the connection 1form of $D^{\hat{b}}$ ) with respect to the covariant derivative $D^{b} A$. Following again Andersson and Dahl's argument [1], and noting that all the imaginaryvalued terms have to cancel out because the left-hand-side of (4.8) is real, one eventually gets: 


$$
\begin{aligned}
& B_{A \nu}\left(\varphi_{u}\right) \\
& =\frac{1}{2} \sum_{i=1}^{n-1}\left(g\left(\left(D_{A \nu}^{b} A\right) e_{i}, A e_{i}\right)-g\left(\left(D_{A e_{i}}^{b} A\right) \nu, A e_{i}\right)\right)\left\langle\varphi_{u}, \varphi_{u}\right\rangle_{g} \\
& \quad+\frac{1}{4} \sum_{i, j, k \text { distinct }} g\left(\left(D_{e_{i}}^{b} A\right) e_{j}, A e_{k}\right)\left\langle c_{g}(A \nu) c_{g}\left(A e_{i}\right) c_{g}\left(A e_{j}\right) c_{g}\left(A e_{k}\right) \varphi_{u}, \varphi_{u}\right\rangle_{g} \\
& \quad+\frac{i}{2} \sum_{i=1}^{n-1}\left\langle c_{g}(A \nu) c_{g}\left(A e_{i}\right) c_{g}\left(A\left(A e_{i}\right)-A e_{i}\right) \varphi_{u}, \varphi_{u}\right\rangle_{g}
\end{aligned}
$$

Using the (spin) isomorphism $A$, this can immediately be rewritten as:

$$
\begin{aligned}
& B_{A \nu}\left(\varphi_{u}\right) \\
& =\frac{1}{2} \sum_{i=1}^{n-1}\left(b\left(A^{-1}\left(D_{A \nu}^{b} A\right) e_{i}, e_{i}\right)-b\left(A^{-1}\left(D_{A e_{i}}^{b} A\right) \nu, e_{i}\right)\right)\left\langle\varphi_{u}, \varphi_{u}\right\rangle_{b} \\
& \quad+\frac{1}{4} \sum_{i, j, k \text { distinct }} b\left(A^{-1}\left(D_{e_{i}}^{b} A\right) e_{j}, e_{k}\right)\left\langle c_{b}(\nu) c_{b}\left(e_{i}\right) c_{b}\left(e_{j}\right) c_{b}\left(e_{k}\right) \varphi_{u}, \varphi_{u}\right\rangle_{b} \\
& \quad+\frac{i}{2} \sum_{i=1}^{n-1}\left\langle c_{b}(\nu) c_{b}\left(e_{i}\right) c_{b}\left(A e_{i}-e_{i}\right) \varphi_{u}, \varphi_{u}\right\rangle_{b},
\end{aligned}
$$

where all computations take now place on the spinor bundle of the reference hyperbolic metric. Taking into account the relationship between the squared norm of $\varphi_{u}$ and $V_{u}$, we now recall that our asymptotic conditions imply that, in the last formula, any quadratic term in $A-I$ and $D^{b} A$ (or, equivalently, in $e=g-b$ ), when integrated on $S_{r}$, has limit value zero as $r$ goes to infinity. One may then eliminate a large number of occurrences of the map $A$ from the above formula. We will use below the notation $U \simeq V$ to mean that $V$ is the only term that contributes when integrating $U$ on larger and larger spheres. Equivalently,

$$
U \simeq V \quad \Longrightarrow \quad \lim _{r \rightarrow \infty} \int_{S_{r}} U=\lim _{r \rightarrow \infty} \int_{S_{r}} V
$$

Then, one obtains in our case: 


$$
\begin{aligned}
B_{A \nu}\left(\varphi_{u}\right) \simeq & \frac{1}{2} \sum_{i=1}^{n-1}\left(b\left(\left(D_{\nu}^{b} A\right) e_{i}, e_{i}\right)-b\left(\left(D_{e_{i}}^{b} A\right) \nu, e_{i}\right)\right)\left\langle\varphi_{u}, \varphi_{u}\right\rangle_{b} \\
& +\frac{1}{4} \sum_{i, j, k \text { distinct }} b\left(\left(D_{e_{i}}^{b} A\right) e_{j}, e_{k}\right)\left\langle c_{b}(\nu) c_{b}\left(e_{i}\right) c_{b}\left(e_{j}\right) c_{b}\left(e_{k}\right) \varphi_{u}, \varphi_{u}\right\rangle_{b} \\
& +\frac{i}{2} \sum_{i=1}^{n-1}\left\langle c_{b}(\nu) c_{b}\left(e_{i}\right) c_{b}\left(A e_{i}-e_{i}\right) \varphi_{u}, \varphi_{u}\right\rangle_{b} .
\end{aligned}
$$

Furthermore, the last term in the previous formula is easily computed and it remains:

$$
\begin{aligned}
B_{A \nu}\left(\varphi_{u}\right) \simeq & \frac{1}{2} \sum_{i=1}^{n-1}\left(b\left(\left(D_{\nu}^{b} A\right) e_{i}, e_{i}\right)-b\left(\left(D_{e_{i}}^{b} A\right) \nu, e_{i}\right)\right)\left\langle\varphi_{u}, \varphi_{u}\right\rangle_{b} \\
& +\frac{1}{4} \sum_{i, j, k \text { distinct }} b\left(\left(D_{e_{i}}^{b} A\right) e_{j}, e_{k}\right)\left\langle c_{b}(\nu) c_{b}\left(e_{i}\right) c_{b}\left(e_{j}\right) c_{b}\left(e_{k}\right) \varphi_{u}, \varphi_{u}\right\rangle_{b} \\
& -\frac{i}{2}\left(\operatorname{tr}(A-I)\left\langle c_{b}(\nu) \varphi_{u}, \varphi_{u}\right\rangle_{b}-\left\langle c_{b}((A-I) \nu) \varphi_{u}, \varphi_{u}\right\rangle_{b}\right) .
\end{aligned}
$$

The second term in this last formula contributes as zero, since $A$ (hence $\left.D^{b} A\right)$ is symmetric and $e_{i} \cdot e_{j}$. is antisymmetric. We then get:

$$
\begin{aligned}
\lim _{r \rightarrow \infty} \int_{S_{r}} B_{A \nu}\left(\varphi_{u}\right)= & \lim _{r \rightarrow \infty} \int_{S_{r}} \frac{1}{2} \sum_{i=1}^{n-1}\left(b\left(\left(D_{\nu}^{b} A\right) e_{i}, e_{i}\right)-b\left(\left(D_{e_{i}}^{b} A\right) \nu, e_{i}\right)\right)\left\langle\varphi_{u}, \varphi_{u}\right\rangle_{b} \\
& -\frac{i}{2}\left(\operatorname{tr}(A-I)\left\langle c_{b}(\nu) \varphi_{u}, \varphi_{u}\right\rangle_{b}-\left\langle c_{b}((A-I) \nu) \varphi_{u}, \varphi_{u}\right\rangle_{b}\right) .
\end{aligned}
$$

To compare with our previous formulae for mass integrals, we now replace $A$ by $I-\frac{1}{2} e$ (as remainder terms in a Taylor expansion contribute as zero in the limit) and relate the norms $\left\langle\varphi_{u}, \varphi_{u}\right\rangle_{b}$ and $\left\langle c_{b}(\nu) \cdot \varphi_{u}, \varphi_{u}\right\rangle_{b}$ to $V_{u}$ using $\left|\varphi_{u}\right|_{b}^{2}=V_{u}$ and $i\left\langle c_{b}(X) \varphi_{u}, \varphi_{u}\right\rangle_{b}=d V_{u}(X)$ to obtain in the limit:

$-\frac{1}{4} \lim _{r \rightarrow \infty} \int_{S_{r}} V_{u}\left(d\left(\operatorname{tr}_{b} e\right)(\nu)-\sum_{i=1}^{n-1} D_{e_{i}}^{b} e\left(\nu, e_{i}\right)\right)-\left(\operatorname{tr}_{b} e\right) d V_{u}(\nu)+d V_{u}(e(\nu))$, or, equivalently,

$$
\lim _{r \rightarrow \infty} \int_{S_{r}} B_{A \nu}\left(\varphi_{u}\right)=\frac{1}{4} \lim _{r \rightarrow \infty} \int_{S_{r}} \mathbb{U}^{i} \nu_{i}
$$

As the left-hand side in the Schrödinger-Lichnerowicz formula (4.11) is nonnegative, this implies that the mass (seen as a linear functional on $\mathcal{N}_{b_{0}}$ ) is nonnegative on any future-directed null vector in $\mathcal{N}_{b_{0}}$. Standard considerations in Lorentzian geometry yield that this linear functional is causal 
and future directed (notice that we use here only the existence of imaginary Killing spinors of Type I, which is valid in any dimension). It remains to show that it is either timelike, or vanishes.

Suppose, then, that the mass $H_{\Phi}$ (still seen as a linear functional on $\mathcal{N}_{b_{0}}$ ) is isotropic, then there exists a nonzero element $W$ of the light cone in $\mathcal{N}_{b_{0}}$ such that it evaluates against $W$ as zero. Up to rescaling, $W$ can be written as $V_{(0)}-\sum_{i} a_{i} V_{(i)}$ and we already noticed there exists a constant spinor $u$ on $(\mathbb{B}, e)$ such that $V_{u}=W$. The Lichnerowicz-Schrödinger formula above has then a vanishing contribution at infinity. This implies the associated spinor $\Phi_{u}$ is a Killing spinor, and H. Baum's work shows that $(M, g)$ is isometric to the hyperbolic space $([\mathbf{1}]$ and $[\mathbf{6}])$. The mass functional $H_{\Phi}$ is then zero and this ends the Proof of Theorem 4.1.

Manifolds with boundary. When $(M, g)$ has a compact boundary one expects that the correct statement is the Penrose inequality $([\mathbf{1 1}, \mathbf{2 6}]$ and $[\mathbf{2 7}])$, which seems to lie outside of the scope of the Witten-type argument given above. Recall, however, that this last argument does lead to a positivity statement $([\mathbf{2 1}, \mathbf{2 2}]$ and $[\mathbf{2 3}])$ when compact boundaries occur:

Theorem 4.7. Let $(M, g)$ be a complete spin manifold with a $C^{2}$ metric, with a compact nonempty boundary of mean curvature

$$
\Theta \leqslant n-1,
$$

and with scalar curvature satisfying

$$
R_{g} \geqslant-n(n-1) \text {. }
$$

If the asymptotic conditions $(2.8)$ and $(2.13)$ hold with $(N, \breve{h})=\left(\mathbb{S}^{n-1}, g_{\mathbb{S}^{n-1}}\right)$, then the covector $p_{(\mu)}$ defined by Equation (3.5) is timelike future directed.

Proof. When $\partial M$ is nonempty, a supplementary boundary integral over $\partial M$, given by

$$
\int_{\partial M} B_{A \nu}\left(\Phi_{u}\right)=\int_{\partial M}\left\langle\mathfrak{D}_{\partial M} \Phi_{u}+\frac{1}{2}\left(\Theta-(n-1) i c_{g}(n)\right) \Phi_{u}, \Phi_{u}\right\rangle,
$$

appears in Equations (4.8) and (4.11), where $\Theta$ is the inwards oriented mean extrinsic curvature of $\partial M$, while $\mathfrak{D}_{\partial M}$ is a boundary Dirac operator. It is defined as

$$
\mathfrak{D}_{\partial M}=c_{g}(n) \sum_{i=1}^{n-1} c_{g}\left(e_{i}\right) \mathcal{D}_{e_{i}}
$$

where $\mathcal{D}$ is the spin connection intrinsic to $\partial M$, explicitly defined on spinors fields on $M$ restricted to $\partial M$ as

$$
\mathcal{D}_{X}=D_{X}-\frac{1}{2} c_{g}(n) c_{g}(B(X)),
$$

where $B$ is the shape operator of the boundary. 
Following [21] (compare [23]) we impose the following boundary condition on the spinors $\Phi_{u}$ :

$$
\Phi_{u}=\varepsilon \Phi_{u}
$$

where $\varepsilon$ is a hermitian involution on spinors given by

$$
\varepsilon=i c_{g}(n)
$$

as in [24]. It is proved in this paper that this leads to a self-adjoint elliptic problem for the Dirac operator which can be solved. Positivity of the mass is obtained through the same argument as before, the boundary contribution (4.23) having the correct sign since $\varepsilon \mathfrak{D}_{\partial M}=-\mathfrak{D}_{\partial M} \varepsilon$ so that

$$
\begin{aligned}
\left\langle\Phi_{u}, \mathfrak{D}_{\partial M} \Phi_{u}\right\rangle & =\left\langle\Phi_{u}, \mathfrak{D}_{\partial M} \varepsilon \Phi_{u}\right\rangle \\
& =-\left\langle\Phi_{u}, \varepsilon \mathfrak{D}_{\partial M} \Phi_{u}\right\rangle \\
& =-\left\langle\varepsilon \Phi_{u}, \mathfrak{D}_{\partial M} \Phi_{u}\right\rangle \\
& =-\left\langle\Phi_{u}, \mathfrak{D}_{\partial M} \Phi_{u}\right\rangle .
\end{aligned}
$$

As a result, $\left\langle\Phi_{u}, \mathfrak{D}_{\partial M} \Phi_{u}\right\rangle$ vanishes and it remains

$$
\int_{\partial M} B_{A \nu}\left(\Phi_{u}\right)=\int_{\partial M}\left\langle\frac{1}{2}(\Theta-(n-1)) \Phi_{u}, \Phi_{u}\right\rangle
$$

for the boundary contribution. This proves as above that the covector $p_{(\mu)}$ defined by Equation (3.5) is timelike future directed, or lightlike future directed, or vanishing. Let us show that those last two possibilities cannot occur: Clearly, $p_{(\mu)}$ can be lightlike or vanish if and only if $M$ carries an imaginary Killing spinor $\Phi_{u}$ satisfying the boundary condition (4.25) at $\partial M$. Further, Equation (4.28) implies that $\Theta$ is identically equal to $(n-1)-$ otherwise, the imaginary Killing spinor field $\Phi_{u}$ would be zero on an open set on the boundary, a situation which is forbidden by the uniqueness property of solutions of ordinary differential equations. Moreover it is a classical fact $[6]$ that existence of an imaginary Killing spinor implies that $(M, g)$ is Einstein, of scalar curvature $-n(n-1)$.

Hence we have the following situation: A noncompact Einstein manifold, looking like the hyperbolic space at infinity and with a compact inner boundary of constant mean curvature $n-1$. Choose any very large sphere-like compact submanifold $S$ in the asymptotically hyperbolic end of $M$ and consider the part of $M$ located inside $S$. It is a compact Einstein $n$-manifold with boundary having two components, one of constant mean curvature $\Theta_{\partial M}=n-1$ and the other one having (not necessarily constant) mean curvature $\Theta_{S}$ close to that of a sphere in the hyperbolic space by (2.13), hence which can be taken so that $\Theta_{S}>n-1$ at each point of $S$ (here, both mean curvatures are computed with respect to the normal unit vector pointing towards infinity). 
Let now $p$ be in $\partial M$ the closest point to $S$ and $\gamma$ be a minimising geodesic from $S$ to $p$, starting from a point $q$ in $S$. Let $\ell$ be the distance from $\partial M$ to $S$, i.e., the distance from $p$ to $q$. We now consider the family $\partial M_{\delta}$ of submanifolds obtained by pushing the boundary $\partial M$ a distance $\delta$ through its normal exponential map towards $S$, and the analogously defined submanifolds $S_{\eta}$ obtained from $S$ by pushing it a distance $\eta$ towards $\partial M$.

For $\delta>0$ small enough, the submanifold $\partial M_{\delta}$ is still smooth. Moreover, the contact point $r$ of $\partial M_{\delta}$ and $\gamma$ is necessarily the closest point of $\partial M_{\delta}$ to $S$ (and is at distance $\ell-\delta$ ). As $\gamma$ is minimising, the distance function to $S$ is smooth in an open neighborhood of $\gamma \backslash\{p\}$, hence the submanifold $S_{\ell-\delta}$ is smooth around $r$, contained in the (closure of the) unbounded part of $M$ delimited by $\partial M_{\delta}$ and is necessarily tangent at $r$ to $\partial M_{\delta}$.

It remains to show that this leads to a contradiction. This follows from classical comparison geometry: The usual Riccati equation for the normalised mean curvature $H=\frac{\Theta}{n-1}$ reads [13]:

$$
H^{\prime} \leqslant-H^{2}-\frac{\operatorname{Ric}\left(\gamma^{\prime}, \gamma^{\prime}\right)}{n-1}
$$

where $H$ stands either for the outwards normalised mean curvature of the family $\left\{\partial M_{\delta}\right\}$ or for the outwards normalised mean curvature of the family $\left\{S_{\eta}\right\}$ and ' denotes differentiation with respect to either $\delta$ or $-\eta$. As $(M, g)$ is Einstein, this translates in our context as:

$$
H^{\prime} \leqslant 1-H^{2}
$$

and one gets by standard arguments that

$$
H_{\partial M_{\delta}} \leqslant 1 \text { and } H_{S_{\eta}}>1 \text { for any } \eta>0, \delta>0 .
$$

At the point $r$, this contradicts the comparison principle for the mean curvature equation, which ends the proof.

Remark 4.8. This result is of special interest in general relativity, where the condition on the mean curvature $(\Theta \leqslant n-1)$ has the following interpretation: Let $\alpha \in \mathbb{R}$ be a constant satisfying $|\alpha| \leq 1$, then our Riemannian manifold can be thought of as arising from a spacelike slice $i(M)$ in a vacuum space-time with cosmological constant $\Lambda:=-\left(1-\alpha^{2}\right) n(n-1) \leq 0$, such that $i(M)$ has extrinsic curvature $K_{i j}=\alpha g_{i j}$. The condition $R_{g} \geq-n(n-1)$ is then equivalent to requiring positive energy density on $i(M)$, while the condition $\Theta \leqslant-\alpha(n-1)$ is equivalent to the statement that $\partial M$ is an outerfuture-trapped, or marginally outer-future-trapped, compact hypersurface in $i(M)$. Under suitable global conditions existence of such surfaces implies existence of a black hole region in the associated space-time. Similarly the condition $\Theta \leqslant \alpha(n-1)$ is associated with outer-past-trapped surfaces, and leads to existence of white hole regions. A significant consequence of the above result is then that the trapped surface situation is far away from 
the case of vanishing mass. This gives mathematical support (disjoint from all known physical reasons) to the idea that some statement analogous to the Penrose inequality ([11] and [27]) should hold in the asymptotically hyperbolic case as well.

Remark 4.9. In the special (and interesting for physics) case $\alpha=0$ (i.e., $\Theta \leq 0$ ), there is a shorter way to prove that mass cannot vanish: In the proof of Theorem 4.7, one may take for $\varepsilon$ any self-adjoint involution satisfying $c_{g}(n) \varepsilon=-\varepsilon c_{g}(n)$ and $\varepsilon \mathfrak{D}_{\partial M}=-\mathfrak{D}_{\partial M} \varepsilon$ (such an $\varepsilon$ will certainly exist if our Riemannian manifold is isometrically embedded as a Riemannian slice in a Lorentzian $(n+1)$-dimensional manifold, or more generally, if the spinor bundle carries a representation of the Clifford algebra of the Lorentzian metric $\gamma=-e_{0} \otimes e_{0}+g$; in any of those cases one sets $\varepsilon=c_{\gamma}\left(e_{0}\right) c_{\gamma}(n)-$ note however that, in the rest of the proof as well as in the other parts of the paper, our discussion will stay purely Riemannian, as opposed to $[\mathbf{2 1}, \mathbf{2 3}]$ and [34]). A calculation identical to (4.27) shows that the boundary integral will have the right sign and the proof goes through without modifications, implying that the covector $p_{(\mu)}$ is timelike future directed, or lightlike future directed, or vanishing. Assuming one of the last two conclusions, the equality case in the Lichnerowicz-Weitzenböck formula yields again existence of an imaginary Killing spinor. When restricted to the boundary, this spinor field would then be an eigenspinor of the formally self-adjoint boundary Dirac operator $\mathfrak{D}_{\partial M}$ for a purely imaginary eigenvalue, which is certainly impossible on a compact manifold.

\section{The mass of conformally compactifiable asymptotically hyperbolic ends.}

The metric $g$ of a Riemannian manifold $(M, g)$ will be said to be $C^{k} c o m$ pactifiable if there exists a compact Riemannian manifold with boundary $\left(\bar{M} \approx M \cup \partial_{\infty} M \cup \partial M, \widetilde{g}\right)$, where $\partial \bar{M}=\partial M \cup \partial_{\infty} M$ is the metric boundary of $(\bar{M}, \widetilde{g})$, with $\partial M$ - the metric boundary of $(M, g)$, together with a diffeomorphism

$$
\psi: \operatorname{int} \bar{M} \rightarrow M
$$

such that

$$
\psi^{*} g=\Omega^{-2} \widetilde{g},
$$

where $\Omega$ is a defining function for $\partial_{\infty} M$ (i.e., $\Omega \geq 0,\{\Omega=0\}=\partial_{\infty} M$, and $d \Omega$ is nowhere vanishing on $\left.\partial_{\infty} M\right)$, with $\widetilde{g}$ - a metric which is $C^{k}$ up-toboundary on $\bar{M}$. The triple $(\bar{M}, \widetilde{g}, \Omega)$ will then be called a $C^{k}$ conformal completion of $(M, g)$. Clearly the definition allows $M$ to have a usual compact boundary. $(M, g)$ will be said to have a conformally compactifiable end $M_{\text {ext }}$ if $M$ contains an open submanifold $M_{\text {ext }}$ (of the same dimension that 
$M)$ such that $\left(M_{\text {ext }},\left.g\right|_{M_{\text {ext }}}\right)$ is conformally compactifiable, with a connected conformal boundary $\partial_{\infty} M_{\text {ext }}$.

In the remainder of this section we shall assume for simplicity that the conformally rescaled metric $\widetilde{g}$ is smooth up to boundary; it should be clear how the conditions here can be adapted to a weighted Hölder or Sobolev setting to allow lower differentiability compactifications consistent with the requirements of Theorem 2.3.

It is easily seen, using the transformation properties of the Riemann tensor under conformal transformations (cf., e.g., [29]) that for smoothly compactifiable metrics all the sectional curvatures $\kappa$ of $g$ satisfy

$$
\left(\kappa+|d \Omega|_{\widetilde{g}^{\#}}^{2}\right)(p) \rightarrow_{p \rightarrow \partial_{\infty} M} 0,
$$

where $|\cdot|_{k}$ denotes the norm of a tensor with respect to a metric $k$; recall that $g^{\#}$ is the metric on $T^{*} M$ associated to $g$.

Now, Equation (5.1) determines only the conformal class $[\widetilde{g}]$ of $\widetilde{g}$. Without loss of generality we can restrict the representative $\widetilde{g}$ of $[\widetilde{g}]$ so that the metric $h_{0}$ induced by $\widetilde{g}$ on $\partial_{\infty} M$ has constant scalar curvature normalised as in (1.3), and this restriction will be made in what follows.

A compactifiable metric will be called asymptotically hyperbolic in an end $M_{\text {ext }}$ if

$$
\forall p \in \partial_{\infty} M_{\text {ext }} \quad|d \Omega|_{\widetilde{g}^{\#}}^{2}(p)=1 .
$$

In what follows we restrict our considerations to a single end $M_{\text {ext }}$, replacing $M$ by $M_{\text {ext }}$ we will assume that $M=M_{\text {ext }}$, so that $\partial_{\infty} M=\partial_{\infty} M_{\text {ext }}$. Whenever (5.3) holds on $\partial_{\infty} M$, a preferred representative of $[\widetilde{g}]$ in a neighborhood of $\partial_{\infty} M$ can be chosen by requiring that

$$
|d \Omega|_{\widetilde{g}^{\#}}^{2} \equiv 1
$$

Using $x:=\Omega$ as the first coordinate, a coordinate system can be constructed (in some perhaps smaller neighborhood of $\partial_{\infty} M$ ) in which $g$ takes the form

$$
g=x^{-2}\left(d x^{2}+h_{x}\right),
$$

where $h_{x}$ is an $x$-dependent family of metrics on $N:=\partial_{\infty} M$. We define the reference metric $b$ as

$$
b:=x^{-2}\left(d x^{2}+\left(1-k x^{2} / 4\right)^{2} h_{0}\right)
$$

(compare Equation (1.8)). To make contact with Section 3, we assume that $b$ is one of the metrics considered there. If $r$ is defined by Equation (1.7), then the asymptotic conditions of Proposition 2.2 and of Theorem 2.3 will hold if and only if

$$
\begin{gathered}
0 \leq i \leq\left.\lfloor n / 2\rfloor \quad \partial_{x}^{i}\left(h_{x}-\left(1-k x^{2} / 4\right)^{2} h_{0}\right)\right|_{x=0}=0 \\
\Longleftrightarrow \quad h_{x}=\left(1-k x^{2} / 4\right)^{2} h_{0}+o\left(x^{\lfloor n / 2\rfloor}\right),
\end{gathered}
$$


where $\lfloor\alpha\rfloor$ denotes the integer value of $\alpha$, and if

$$
R_{g}+n(n-1)=O\left(x^{n-1}\right) .
$$

For instance, in the physically significant case $n=3$, Equation (5.6) is equivalent to the requirement that the second fundamental form of $\partial_{\infty} M$ vanishes in the conformal gauge (5.4).

Under Equations (5.6)-(5.7), given some compactification $\left(\bar{M}_{1}, g_{1}, \Omega_{1}\right)$ of an end $\left(M_{\text {ext }}, g\right)$ of $M$, we use the background (5.5) to define its mass, whenever the resulting background is one of those discussed in Section 3 . (As already pointed out, when $\left(\partial_{\infty} M, h_{0}\right)=\left(\mathbb{S}^{n-1}, g_{\mathbb{S}^{n-1}}\right)$ this definition coincides with that of [39].) Consider a second compactification $\left(\bar{M}_{2}, g_{2}, \Omega_{2}\right)$ of $(M, g)$ satisfying the above requirements; it is far from clear that the resulting mass will be the same. This turns out to be the case:

Theorem 5.1. Suppose that $(M, g)$ contains a conformally compactifiable end $M_{\text {ext }}$ such that $\left(\partial_{\infty} M_{\text {ext }},\left.\widetilde{g}\right|_{\partial_{\infty} M_{\text {ext }}}\right)$ is one of the manifolds considered in Section 3 (that is, the scalar curvature of $\breve{h}$ is as in (1.3) and either $\breve{h}$ has strictly negative Ricci curvature, or is flat, or is the round sphere, or a quotient thereof). Assume, moreover, that Equations (5.6)-(5.7) hold. Then the mass of $M_{\mathrm{ext}}$, as defined above, is independent of the compactification of $M_{\text {ext }}$ chosen for its calculation.

Proof. As already pointed out above, we can modify the $g_{a}$ 's and $\Omega_{a}$ 's so that

$$
\left|d \Omega_{a}\right|_{g_{a}^{\#}}=1
$$

in a neighborhood of $\partial_{\infty} M_{a}$. Using the $\Omega_{a}$ 's as the first coordinate, in neighborhoods of respective boundaries we can write the metrics $g_{a}$ as

$$
g_{a}=d \Omega_{a}^{2}+h^{a},
$$

where $h^{a}$ is the metric induced by $g_{a}$ on the level sets of $\Omega_{a}$. We introduce radial coordinates $r_{a}$ as in (1.7),

$$
r_{a}=\frac{1-k \Omega_{a}^{2} / 4}{\Omega_{a}}
$$

so that

$$
g=\frac{d r_{1}^{2}}{r_{1}^{2}+k}+r_{1}^{2} h_{A B}^{1} d v_{1}^{A} d v_{1}^{B}=\frac{d r_{2}^{2}}{r_{2}^{2}+k}+r_{2}^{2} h_{A B}^{2} d v_{2}^{A} d v_{2}^{B},
$$

where $k$ is defined by Equation (1.3) using the boundary metric arising out from $g_{1}$, and we have denoted by $\left(r_{a}, v_{a}^{A}\right), a=1,2$, the corresponding local coordinates near $\partial_{\infty} M_{a}$. It follows from [16, Theorem 3.3] that the map

$$
\left(r_{1}, v_{1}^{A}\right) \rightarrow\left(r_{2}, v_{2}^{A}\right)
$$


extends by continuity to a differentiable map from $\bar{M}_{1}$ to $\bar{M}_{2}$. Equivalently, $\phi_{1}^{-1} \circ \phi_{2}$ extends by continuity to a continuous map from $\bar{M}_{1}$ to $\bar{M}_{2}$. Further, point 1 of [16, Theorem 3.3] shows that the limit

$$
\lim _{r_{1} \rightarrow \infty} v_{2}^{A}\left(r_{1}, v_{1}^{A}\right)
$$

exists, and defines a $C^{\infty}$ conformal diffeomorphism $\Psi$ from $\left(\partial_{\infty} M_{1},\left.h^{1}\right|_{\partial_{\infty} M_{1}}\right)$ to $\left(\partial_{\infty} M_{2},\left.h^{2}\right|_{\partial_{\infty} M_{2}}\right)$ :

$$
\left.\Psi^{*} h^{2}\right|_{\partial_{\infty} M_{2}}=\left.e^{\psi} h^{1}\right|_{\partial_{\infty} M_{1}} .
$$

Replacing $g_{1}$ by $e^{\psi} g_{1}$ and $\Omega_{1}$ by $\Omega_{1} e^{\psi / 2}$, where, by an abuse of notation, we use the same symbol $e^{\psi}$ to denote the extension of $e^{\psi}$ from $\partial_{\infty} M_{1}$ to $\bar{M}_{1}$ such that

$$
\left|d\left(\Omega_{1} e^{\psi / 2}\right)\right|_{\left(e^{\psi} g\right) \#}=1,
$$

we obtain Equation (5.9) with $\psi=0$, hence $\left.h^{1}\right|_{\partial_{\infty} M_{1}}$ is isometric to $\left.h^{2}\right|_{\partial_{\infty} M_{2}}$. As a result, Theorem 2.3 together with the discussion of Section 3 establishes Theorem 5.1.

We note that the argument just given also proves the following:

Proposition 5.2. Consider $\left(M_{\mathrm{ext}}, b\right)$ with a metric of the form (1.2). Then for every conformal isometry $\Psi$ of $(N, \breve{h})$ there exists $R_{*}>0$ and a bisometric map $\Phi:\left[R_{*}, \infty\right) \times N \rightarrow[R, \infty) \times N$, such that

$$
\lim _{r \rightarrow \infty} \Phi(r, \cdot)=\Psi(\cdot) \text {. }
$$

\section{Uniqueness of conformal completions.}

It should be clear that the invariance of the mass is related to the question of uniqueness of conformal compactifications. There are several issues to address here: $g_{1}$ is conformal to an appropriate pull-back of $g_{2}$ on the interior of $\bar{M}_{1}$, but the relative conformal factor could blow up as one approaches the boundary of $M_{1}$. Even if the relative conformal factor remains uniformly bounded both from above and below, it is not clear whether or not it extends differentiably — or even just continuously — to the boundary. Let us show that things behave as expected, so that conformal completions are conformally diffeomorphic in the sense of manifolds with boundary; notations are the same as in the previous section.

Theorem 6.1. Let $(M, g)$ be a Riemannian manifold endowed with two $C^{\infty}$-conformal compactifications $\left(\bar{M}_{1}, g_{1}, \Omega_{1}\right)$ and $\left(\bar{M}_{2}, g_{2}, \Omega_{2}\right)$. Then

$$
\phi_{1}^{-1} \circ \phi_{2}: \operatorname{int} M_{2} \rightarrow \operatorname{int} M_{1}
$$

extends by continuity to a $C^{\infty}$ conformal up-to-boundary diffeomorphism from $\left(\bar{M}_{2}, g_{2}\right)$ to $\left(\bar{M}_{1}, g_{1}\right)$, in particular $\bar{M}_{1}$ and $\bar{M}_{2}$ are diffeomorphic as manifolds with boundary. 
Remark 6.2. Results about completions of finite differentiability can be obtained in a similar way, by chasing the order of differentiability through various steps of the arguments below.

Proof. Let $\varphi_{2}$ be defined on $\operatorname{int} \bar{M}_{1}$ by the equation

$$
\varphi_{2}:=\frac{\Omega_{2} \circ \phi_{2}^{-1} \circ \phi_{1}}{\Omega_{1}}>0
$$

Equation (5.8) gives

$$
\begin{aligned}
1 & =\left|d \Omega_{2}\right|_{g_{2}^{\#}}^{2} \\
& =\left|\varphi_{2} d \Omega_{1}+\Omega_{1} d \varphi_{2}\right|_{g_{2}^{\#}}^{2} \\
& =\varphi_{2}^{2}\left|d \Omega_{1}\right|_{g_{2}^{\#}}^{2}+2 \varphi_{2} \Omega_{1} g_{2}^{\#}\left(d \varphi_{2}, d \Omega_{1}\right)+\Omega_{1}^{2}\left|d \varphi_{2}\right|_{g_{2}^{\#}}^{2} \\
& =\left|d \Omega_{1}\right|_{g_{1}^{\#}}^{2}+2 \varphi_{2}^{-1} \Omega_{1} g_{1}^{\#}\left(d \varphi_{2}, d \Omega_{1}\right)+\varphi_{2}^{-2} \Omega_{1}^{2}\left|d \varphi_{2}\right|_{g_{1}^{\#}}^{2},
\end{aligned}
$$

hence

$$
2 \Omega_{1} g_{1}^{\#}\left(d\left(\ln \varphi_{2}\right), d \Omega_{1}\right)=-\Omega_{1}^{2}\left|d\left(\ln \varphi_{2}\right)\right|_{g_{1}^{\#}}^{2} \leq 0 .
$$

We can identify a neighborhood of $\partial M_{1}$ with $\partial M_{1} \times\left[0, x_{0}\right]$ using the flow of $g_{1}^{\#}\left(d \Omega_{1}, \cdot\right)$. Equation (6.2) shows that $\ln \varphi_{2}$ is monotonously increasing along the integral curves of the vector field $g_{1}^{\#}\left(d \Omega_{1}, \cdot\right)$ when $\Omega_{1}$ decreases, so that there exists a constant $C_{2}:=\inf _{\partial M_{1} \times\left\{x_{0}\right\}} \varphi_{2}$ such that on $\partial M_{1} \times\left[0, x_{0}\right]$ we have

$$
\varphi_{2} \geq C_{2}>-\infty
$$

Applying the same argument, with $g_{1}$ and $g_{2}$ interchanged, to

$$
\varphi_{1}:=\frac{\Omega_{1} \circ \phi_{1}^{-1} \circ \phi_{2}}{\Omega_{2}}=\frac{1}{\varphi_{2}} \circ \phi_{1}^{-1} \circ \phi_{1}: \operatorname{int} \bar{M}_{2} \rightarrow \mathbb{R}
$$

shows that on $\partial M_{1} \times\left[0, x_{0}\right]$ it holds

$$
\varphi_{1} \geq C_{1}>-\infty
$$

Equations (6.3) and (6.4) clearly imply that the $\varphi_{a}$ 's are uniformly bounded and uniformly bounded away from zero.

Set

$$
\phi_{12}:=\phi_{1}^{-1} \circ \phi_{2}, \quad \phi_{21}:=\phi_{2}^{-1} \circ \phi_{1} .
$$


Let $\sigma_{g_{a}}$ denote the distance function associated with the metric $g_{a}$. For $p, q$ in $\operatorname{int} \bar{M}_{1}$ we have

$$
\begin{aligned}
\sigma_{g_{2}}\left(\phi_{21}(p), \phi_{21}(q)\right) & =\inf _{\Gamma} \int_{\Gamma} \sqrt{g_{2}\left(\frac{d \Gamma}{d s}, \frac{d \Gamma}{d s}\right)} \\
& =\inf _{\Gamma} \int_{\Gamma} \sqrt{\varphi_{1}^{-2}\left(\phi_{12}^{*} g_{1}\right)\left(\frac{d \Gamma}{d s}, \frac{d \Gamma}{d s}\right)} \\
& \geq \inf _{\Gamma} C \int_{\Gamma} \sqrt{\left(\phi_{12}^{*} g_{1}\right)\left(\frac{d \Gamma}{d s}, \frac{d \Gamma}{d s}\right)} \\
& =C \inf _{\phi_{12}(\Gamma)} \int_{\phi_{12}(\Gamma)} \sqrt{g_{1}\left(\frac{d \phi_{12}(\Gamma)}{d s}, \frac{d \phi_{12}(\Gamma)}{d s}\right)} \\
& =C \sigma_{g_{1}}(p, q) .
\end{aligned}
$$

This, together with an identical calculation with $g_{1}$ and $g_{2}$ interchanged shows that $\phi_{12}$ and $\phi_{21}$ are uniformly Lipschitz continuous.

Clearly, $\bar{M}_{2}$ is the metric completion of the manifold $M$ with respect to the metric $\left(\phi_{2}^{-1}\right)^{*} g_{2}$; similarly for $\bar{M}_{1}$. An identical calculation shows that the metrics $\left(\phi_{a}^{-1}\right)^{*} g_{a}, a=1,2$ define uniformly equivalent distance functions. But completions obtained using equivalent distances are homeomorphic; it follows that $\bar{M}_{1}$ is homeomorphic to $\bar{M}_{2}$, in particular $\partial M_{1}$ is homeomorphic to $\partial M_{2}$. In fact, by definition we have

$$
\phi_{21} \circ \phi_{12}=\mathrm{id}_{M_{2}}, \quad \phi_{12} \circ \phi_{21}=\mathrm{id}_{M_{1}} .
$$

Since $\phi_{12}$ and $\phi_{21}$ are continuous, they have an extension by continuity to the metric completed spaces; we will use the same symbol to denote those extensions. It is then easily seen that (6.6) with $M_{a}$ replaced by $\bar{M}_{a}$ holds for the extensions, so that the extensions do directly provide the desired homeomorphism. Equation (6.5), together with its equivalent with $g_{1}$ interchanged with $g_{2}$, further show that the extensions $\phi_{21}$ and $\phi_{12}$ are uniformly Lipschitz continuous on $\bar{M}_{1}$ and $\bar{M}_{2}$. Obviously

$$
\phi_{21}: \partial M_{1} \rightarrow \partial M_{2}, \quad \phi_{12}: \partial M_{2} \rightarrow \partial M_{1},
$$

with $\left.\phi_{21}\right|_{\partial M_{1}}$ and $\left.\phi_{12}\right|_{\partial M_{2}}$ being homeomorphisms inverse to each other by the completed spaces equivalent of (6.6). We have:

Lemma 6.3. The map $\phi_{21}$ is $C^{1}$ up-to-boundary.

Proof. We can conformally rescale $g$ so that (5.3) holds with $\widetilde{g}=g_{1}$ and $\Omega=\Omega_{1}$; as (5.3) is conformally invariant, (5.3) will also hold with $\widetilde{g}=g_{2}$ and $\Omega=\Omega_{2}$. Introducing coordinates $r_{a}$ as in the proof of Theorem 5.1 with, say $k=0$, we can apply Theorem 3.3 of [16] to obtain the desired conclusion. 
Returning to the proof of Theorem 6.1, Lemma 6.3 shows that for all $p \in \partial M_{1}$ the maps $\left(\phi_{21}\right)_{*}(p)$ are similarities with nonzero ratio (in general depending upon $p$ ). Differentiability of $\phi_{21}$ further implies that $\phi_{21}$ is $\mathrm{ACL}^{n}$, as defined in [31]. We can then use a deep result of Lelong-Ferrand [31, Theorem A] to conclude that $\left.\varphi_{2}\right|_{\partial M_{1}}$ and $\left.\phi_{21}\right|_{\partial M_{1}}$ are smooth. Now, $u:=$ $\left(\varphi_{2}\right)^{\frac{n-2}{2}}$ solves the Yamabe equation,

$$
\Delta_{g_{1}} u-\frac{n-2}{4(n-1)} R_{g_{1}} u=\left(R_{g_{2}} \circ \phi_{21}\right) u^{(n+2) /(n-2)} .
$$

Here, as before, $R_{g_{a}}$ denotes the curvature scalar of the metric $g_{a}$. The right-hand-side of this equation is in $L^{\infty}\left(\bar{M}_{1}\right)$, and standard results on the Dirichlet problem imply that $u$ - and hence $\varphi_{2}$ - is uniformly $C^{1}$ on $\bar{M}_{1}$. Now, $\phi_{21}$ is an isometry between $g_{1}$ and $\varphi_{2}^{-2} g_{2}$ which implies that, in local coordinates, $\phi_{21}$ satisfies on $M_{1}$ the over-determined set of equations

$$
\frac{\partial^{2} \phi_{21}^{i}}{\partial x^{\ell} \partial x^{m}}=\Gamma_{\ell m}^{k}(x) \frac{\partial \phi_{21}^{i}}{\partial x^{k}}-\bar{\Gamma}_{r s}^{i}\left(\phi_{21}(x)\right) \frac{\partial \phi_{21}^{r}}{\partial x^{\ell}} \frac{\partial \phi_{21}^{s}}{\partial x^{m}},
$$

where $\bar{\Gamma}_{r s}^{i}$ are, in local coordinates, the Christoffel symbols of the Riemannian metric $\left(\varphi_{2} \circ \phi_{12}\right)^{2} g_{2}$. The right-hand-side of this set of equations extends by continuity to a continuous function on $\bar{M}_{1}$, which shows that $\phi_{21}$ is uniformly $C^{2}$ on $\bar{M}_{1}$. It follows that the right-hand-side of Equation (6.7) is uniformly $C^{1}$ on $\bar{M}_{1}$, hence $\varphi_{2}$ is uniformly $C^{2}$ on $\bar{M}_{1}$. An inductive repetition of this argument establishes our claims.

Remark 6.4. It would be of interest to find a proof of Lemma 6.3 which is more in the spirit of conformal geometry than the methods of [16].

Remark 6.5. Recall that there exists a conformally invariant version of the Abbott-Deser mass, due to Ashtekar and Magnon [2], in a Lorentzian spacetime setting. We expect this expression to have a Riemannian counterpart which is also conformally invariant, with the numerical value thereof identical to that of the expression we propose. If that is the case, Theorem 5.1 is actually a straightforward corollary of Theorem 6.1; in particular one would not need to invoke the rather messy calculations of Theorem 2.3, which are implicitly used in the proof of Theorem 5.1.

Acknowledgements. The authors are grateful to Maung Min-Oo and Abdelghani Zeghib for useful discussions and to the referee for his comments which led to an improved version of Theorem 4.7. PTC wishes to thank the Albert Einstein Institute, Golm, for friendly hospitality during part of the work on this paper. 


\section{References}

[1] L. Andersson and M. Dahl, Scalar curvature rigidity for asymptotically locally hyperbolic manifolds, Ann. Global Anal. Geom., 16 (1998), 1-27, MR 99d:53035, Zbl 0946.53021.

[2] A. Ashtekar and A. Magnon, Asymptotically anti-de Sitter space-times, Class. Quantum Grav., 1 (1984), L39-L44, MR 85h:83029.

[3] M. Bañados, C. Teitelboim and J. Zanelli, Black hole in three-dimensional spacetime, Phys. Rev. Lett., 69 (1992), 1849-1851, MR 93e:83042, Zbl 0968.83514.

[4] R. Bartnik, The mass of an asymptotically flat manifold, Comm. Pure Appl. Math., 39 (1986), 661-693, MR 88b:58144, Zbl 0598.53045.

[5] R. Bartnik and P.T. Chruściel, Spectral boundary conditions for Dirac-type equations, in preparation.

[6] H. Baum, Complete Riemannian manifolds with imaginary Killing spinors, Ann. Glob. Anal. Geom., 7 (1989), 205-226, MR 91k:58130, Zbl 0694.53043.

[7] _ Odd-dimensional Riemannian manifolds with imaginary Killing spinors, Ann. Glob. Anal. Geom., 7 (1989), 141-154, MR 91b:53055, Zbl 0708.53039.

[8] A.L. Besse, Einstein Manifolds, Ergebnisse der Mathematik und ihrer Grenzgebiete. 3. Folge, Vol. 10, Springer Verlag, Berlin, New York, Heidelberg, 1987, MR 88f:53087, Zbl 0613.53001.

[9] W. Boucher, G.W. Gibbons and G.T. Horowitz, Uniqueness theorem for anti-de Sitter spacetime, Phys. Rev., D30 (1984), 2447-2451, MR 86e:83014.

[10] J-P. Bourguignon and P. Gauduchon, Spineurs, opérateurs de Dirac et variations de métriques, Comm. Math. Phys., 144 (1992), 581-599, MR 93h:58164, Zbl 0755.53009.

[11] H. Bray, Proof of the Riemannian Penrose conjecture using the positive mass theorem, J. Differential Geom., 59 (2001), 177-267, CMP 1908823.

[12] D. Brill, J. Louko and P. Peldan, Thermodynamics of $(3+1)$-dimensional black holes with toroidal or higher genus horizons, Phys. Rev., D56 (1997), 3600-3610, MR 98i:83047.

[13] J. Cheeger, Critical points of distance functions and applications to geometry, Geometric topology: Recent developments (Montecatini Terme, 1990), Lect. Notes in Math., 1504, Springer, 1992, 1-38, MR 94a:53075, Zbl 0771.53015.

[14] Y. Choquet-Bruhat, Positive-energy theorems, Relativity, groups and topology, II (Les Houches, 1983) (B.S. deWitt and R. Stora, eds.), North-Holland, Amsterdam, 1984, 739-785, MR 87g:83054, Zbl 0593.53055.

[15] P.T. Chruściel, Boundary conditions at spatial infinity from a Hamiltonian point of view, Topological Properties and Global Structure of Space-Time (P. Bergmann and V. de Sabbata, eds.), Plenum Press, New York, 1986, 49-59, MR 92h:83003, Zbl 0687.53070.

[16] P.T. Chruściel and G. Nagy, The mass of spacelike hypersurfaces in asymptotically anti-de Sitter space-times, Adv. Theor. Math. Phys., 5 (2001), 697-754, CMP 1926293.

[17] P.T. Chruściel and W. Simon, Towards the classification of static vacuum spacetimes with negative cosmological constant, Jour. Math. Phys., 42 (2001), 1779-1817, MR 2002j:83013, Zbl 1009.83009. 
[18] E. Delay, Analyse précisée d'équations semi-linéaires elliptiques sur l'espace hyperbolique et application à la courbure scalaire conforme, Bull. Soc. Math. France, 125 (1997), 345-381, MR 99j:53045, Zbl 0939.53026.

[19] T. Friedrich and I. Kath, Einstein manifolds of dimension five with small first eigenvalue of the Dirac operator, J. Differential Geom., 29 (1989), 263-279, MR 90e:58158, Zbl 0633.53069.

[20] P. Gauduchon, Hermitian connections and Dirac operators, Bolletino U.M.I., 11 B (1997), 257-288, MR 98c:53034, Zbl 0876.53015.

[21] G.W. Gibbons, S.W. Hawking, G.T. Horowitz and M.J. Perry, Positive mass theorem for black holes, Comm. Math. Phys., 88 (1983), 295-308, MR 84k:83015.

[22] M. Herzlich, A Penrose-like inequality for the mass of Riemannian asymptotically flat manifolds, Comm. Math. Phys., 188 (1997), 121-133, MR 99a:53039, Zbl 0886.53032.

[23] _ The positive mass theorem for black holes revisited, J. Geom. Phys., 26 (1998), 97-111, MR 99g:83029, Zbl 0947.83022.

[24] O. Hijazi, S. Montiel and X. Zhang, Conformal lower bounds for the Dirac operator of embedded hypersurfaces, Asian J. Math., 6 (2002), 23-36, MR 2003i:58058.

[25] G.T. Horowitz and R.C. Myers, The AdS/CFT correspondence and a new positive energy conjecture for general relativity, Phys. Rev., D59 (1999), 026005 (12 pp.), MR 2000b:83020.

[26] G. Huisken and T. Ilmanen, The Riemannian Penrose inequality, Internat. Math. Res. Notices, 20 (1997), 1045-1058, MR 98m:53055, Zbl 0905.53043.

[27] _ The inverse mean curvature flow and the Riemannian Penrose inequality, J. Differential Geom., 59 (2001), 353-437, MR 2003h:53091.

[28] F. Kottler, Über die physikalischen Grundlagen der Einsteinschen Gravitationstheorie, Annalen der Physik, 56 (1918), 401-462, Zbl 46.1306.01.

[29] W. Kuehnel, Conformal transformations between Einstein spaces, Conformal geometry (R.S. Kulkarni and U. Pinkall, eds.), Aspects Math.: E, 12, F. Vieweg \& Sohn, Braunschweig, 1988, 105-146, Zbl 0667.53039.

[30] C. LeBrun, Counterexamples to the generalized positive action conjecture, Comm. Math. Phys., 118 (1988), 591-596, MR 89f:53107, Zbl 0659.53050.

[31] J. Lelong-Ferrand, Geometrical interpretations of scalar curvature and regularity of conformal homeomorphisms, Differential geometry and relativity, Mathematical Phys. and Appl. Math., 3, Reidel, Dordrecht, 1976, 91-105, MR 56 \#1222, Zbl 0345.53028.

[32] P. Li and L.-F. Tam, Complete surfaces with finite total curvature, J. Differential Geom., 33 (1991), 139-168, MR 92e:53051, Zbl 0749.53025.

[33] A. Lichnerowicz, Spineurs harmoniques, C.R. Acad. Sci. Paris Sér. A-B, 257 (1963), 7-9, MR 27 \#6218, Zbl 0136.18401.

[34] M. Min-Oo, Scalar curvature rigidity of asymptotically hyperbolic spin manifolds, Math. Ann., 285 (1989), 527-539, MR 91b:53047, Zbl 0686.53038.

[35] T. Parker and C. Taubes, On Witten's proof of the positive energy theorem, Comm. Math. Phys., 84 (1982), 223-238, MR 83m:83020, Zbl 0528.58040.

[36] E. Schrödinger, Diracsches Elektron im Schwerfeld, Preuss. Akad. Wiss. Phys.-Math., 11 (1932), 436-460, Zbl 0004.28100.

[37] K. Shiohama, Total curvature and minimal area of complete surfaces, Proc. Amer. Math. Soc., 94 (1985), 310-316, MR 86h:53047, Zbl 0582.53036. 
[38] L. Vanzo, Black holes with unusual topology, Phys. Rev., D56 (1997), 6475-6483, MR 98i:83054.

[39] X. Wang, Mass for asymptotically hyperbolic manifolds, J. Differential Geom., 57 (2001), 273-299, MR 2003c:53044.

[40] E. Witten, A simple proof of the positive energy theorem, Comm. Math. Phys., 80 (1981), 381-402, MR 83e:83035.

[41] X. Zhang, A definition of total energy-momenta and the positive mass theorem on asymptotically hyperbolic 3 manifolds I. Preprint, 2001.

Received October 2, 2001 and revised March 7, 2003. The first author is supported in part by the Polish Research Council grant KBN 2 P03B 073 15. The second author is a member of the EDGE Research Training Network HPRN-CT-2000-00101 of the European Union and is also supported in part by the ACI program of the French Ministry of Research.

DÉPARTEMENT DE MAThÉmatiques

UMR 6083 DU CNRS

Université DE TOURS

PARC DE GRANDMONT

F-37200 TOURS

FRANCE

E-mail address: chrusciel@univ-tours.fr

Institut De Mathématiques et Modélisation De Montpellier

UMR 5030 DU CNRS

Université Montpellier II

F-34095 Montpellier Cedex 5

FRANCE

E-mail address: herzlich@math.univ-montp2.fr 